\title{
Mass and infrared regularization in a nonlightlike axial gauge
}

\author{
B. Humpert*
}

Swiss Federal Institute of Technology, Laboratory of High Energy Physics, CH-5234 Villigen, Switzerland

\author{
W. L. van Neerven ${ }^{\dagger}$ \\ National Institute for Nuclear and High Energy Physics, NIKHEF-H, P.O. Box 41882, Amsterdam, The Netherlands
}

(Received 20 April 1981)

\begin{abstract}
The complete order-g ${ }^{2}$ deep-inelastic (DI) and Drell-Yan (DY) parton cross sections are evaluated in a general $\left(n^{2} \neq 0\right)$ axial gauge. Our study tests the compatibility of the principal-value prescription with basic field-theory principles (unitarity, analyticity, and gauge invariance), and it confirms the validity of the known on/off-shell mass and infrared regularization techniques, provided the double-cut rule is followed. By focusing on the nonlogarithm terms, we go beyond all earlier such studies and indeed analyze the most sensitive contributions. Our results give explicit insight into the gauge dependence of the mass and infrared singularities and exhibit their cancellation mechanisms. A suitable choice of $n^{2}$ permits the determination of the nonlogarithm asymptotic-freedom corrections in the DY process. We explain how the arising technical difficulties are resolved.
\end{abstract}

\section{INTRODUCTION}

Gauge field theories bear the freedom of gauge choice. In a non-Abelian gauge field theory like quantum chromodynamics (QCD), the choice of a covariant gauge leads to Faddeev-Popov ghosts which, however, decouple from the physical $S$ matrix. In order to prevent the ghost problem, a gauge can be chosen where the gauge field has only physical polarizations. Since the discovery of QCD as a theory of strong interactions, much interest has been devoted to the axial gauge $(n \cdot A=0),{ }^{1}$ which is an example of a ghost-free gauge. The gauge field (gluon) propagator is then given by

$$
\begin{aligned}
D^{\mu \nu}(k) & =\frac{-i}{k^{2}+i \epsilon}\left[g^{\mu \nu}-\frac{n^{\mu} k^{\nu}+n^{\nu} k^{\mu}}{n \cdot k}+n^{2} \frac{k^{\mu} k^{\nu}}{(n \cdot k)^{2}}\right] \\
& \equiv \frac{-i}{k^{2}+i \epsilon} P^{\mu \nu} .
\end{aligned}
$$

The axial gauge is most fruitful in establishing formal proofs, for instance, of mass factorization in deep-inelastic (DI) processes. In the calculation of Green's functions and/or cross sections, however, extra complexities arise due to the spurious singularities $(n \cdot k)^{-a}$ in the second and third terms of Eq. (1.1). In a gauge-invariant set of graphs the spurious singularities are expected to cancel. In explicit calculations, considerable simplifications are achieved if the lightlike $\left(n^{2}=0\right)$ gauge is chosen. The last term in $\mathrm{Eq}$. (1.1) disappears and the integrals containing the $(n \cdot k)^{-1}$ term reveal the structure of those familiar from the covariant gauges. ${ }^{3}$ While evaluating the Feynman integral, the $k=0$ singularity is prevented by a regularization prescription ${ }^{1}$ with all regularized terms being canceled in the sum of a gauge-invariant set of Feynman diagrams. Pursuing the calculation $n^{2} \neq 0$ leads to considerable mathematical complexities - already in lowest order. Earlier analyses have limited themselves to the leading logarithmic terms of the DI structure functions and Green's functions. ${ }^{1,4-6}$ There is no investigation which has pushed the analysis to the highly sensitive (and indeed decisive) nonlogarithm contributions. We emphasize their importance since they are intimately related to the asymptotic-freedom (AF) correction terms of the DI structure functions and the Drell-Yan (DY) cross section.

The subsequent investigation pays particular attention to a set of problems which are briefly mentioned here. The first problem concerns the $(n \cdot k)^{-a}$ singularities which are usually defined in a distribution sense. Several different definitions are equally possible, leading to nonunique Green's functions. ${ }^{6}$ In this study we limit ourselves to the principal-value prescription ${ }^{1}$ which is defined by

$$
\frac{1}{(n \cdot k)^{a}} \Rightarrow \lim _{\epsilon \rightarrow 0} \frac{1}{2}\left[\frac{1}{(n \cdot k+i \epsilon)^{a}}+\frac{(-)^{a}}{(-n \cdot k+i \epsilon)^{a}}\right] \text {. }
$$

Although successfully tested in a few low-order calculations (limited to logarithms only, ${ }^{4-6}$ its validity for all orders in perturbation theory is undecided. The second problem is connected with the appearance of new mass and infrared singularities due to the second and third terms in Eq. (1.1) which also give rise to the (axial) gauge singularity at $n^{2}=0$. The utility of the familiar mass (M) and infrared (IR) regularization techniques, ${ }^{7}$ in particular the on/off-shell methods, must therefore be doubted and need to be rechecked. Our third problem concerns analyticity and unitarity. Although unitarity was generally proved to be satisfied by the axial gauge, ${ }^{1}$ its explicit verification on individual Feynman diagrams using the Cutkosky rules and dispersion techniques is by no means trivial. The gauge parameter $n$, as well 
as the changed $M /$ IR structure of the individual Feynman diagrams, lead to unfamiliar singularities which have to be treated with care. With a fourth question we ask: do the DI/DY coefficient functions actually remain gauge independent? ${ }^{8}$ This problem recently came up in a discussion on the conventional off-shell mass regularization ${ }^{9}$ and, in particular, its applicability to determine the nonleading AF corrections to the DY process. The correct evaluation of the nonlogarithm terms in the DI/DY (axial gauge) parton cross sections is here of prime importance, whereby in both processes the same $n$ value must be maintained. This problem has so far not been satisfactorily studied.

This paper presents a systematic evaluation of all $O\left(g^{2}\right)$ DI and DY cut graphs (Feynman diagrams with unitarity cut $\rightarrow$ cross section) contributing to the processes $\gamma^{*} q \rightarrow g q$ (DI) and $q \bar{q} \rightarrow \gamma^{*} g$ (DY). A nonlightlike $\left(n^{2} \neq 0\right)$ axial gauge is used, and the nonlogarithm contributions to the parton cross sections are given special attention. Our study is an extension of earlier work in this direction. ${ }^{7,10}$. We aim at a complete comparison between axial and Feynman gauges, the latter being representative of all covariant gauges.

This paper is divided into six sections: Sec. II is primarily concerned with the technical side of our study; it specifies $n$, exposes how renormalization is carried out, and discusses the used mass assignments. In Sec. III our results for the DI parton structure function $\digamma_{2}\left(z, q^{2}\right)$ are presented, and analogously Sec. IV is concerned with the DY parton cross section $W\left(z, q^{2}\right)$. Our conclusion and summary are in Sec. VI. The Appendices give calculational details and our results are shown in Tables I-IX.

\section{CALCULATION PROCEDURE}

Before going into the details of our calculations, we discuss in this section the crucial assumptions in our calculations and explain the techniques employed.

Our first discussion point concerns the vector $n^{\mu}$. It can be freely chosen, but once a choice is made it must be consistently followed. Calculations with unspecified $n^{2}$ are awkward. In this paper we have chosen $n$ spacelike $\left(n^{2}<0\right)$. Considerable simplifications occur if $n^{2}$ is identified with an asymptotic kinematical variable, which in the Bjorken limit becomes larger than the regulator masses. In our analysis of the DI graphs we identify $n=-q$, whereas in the DY process we set $n=p_{1}-p_{2}$. If the gluon momentum is ignored, $n^{\mu}$ has one and the same value.' Such an approximation, however, cannot be applied to the hard-gluon $(z=1)$ contributions where the above DI/DY choices for $n^{\mu}$ clearly differ. Our subsequent calculations reveal, however, that in the sum of the hard-gluon contributions all $n$ dependence drops out as can be verified by considering $\mathcal{F}^{R \text {-hard }}$ in DI scattering $[\mathrm{Eq} .(3.26)]$ and $W^{R \text {-hard }}$ in the DY process [Eq. (4.8)]. Therefore, $n$ in DI scattering is allowed to be different from $n$ in the DY process as far as the hard-gluon part is concerned. Since in the soft/virtual-gluon contributions all dependence on the (final state) gluon momentum drops out, the DI $n^{\mu}$ reduces to $n$ $=p_{1}-p_{2}$ (remember $q=p_{2}+k-p_{1}$ in DI scattering) and agrees with the DY choice. Furthermore, the virtual- and soft-gluon contributions to the DI structure function and the DY cross section depend exclusively on the variables $q^{2}, n^{2}, n \cdot p_{1}$, $n \cdot p_{2}$. Choosing $q^{2}=-Q^{2}$, their numerical values remain unchanged while going from the spacelike to the timelike region (for $n^{2}=-Q^{2}$ we have $n \cdot p_{1}$ $\left.=-Q^{2} / 2, n \cdot p_{2}=+Q^{2} / 2\right)$. Note finally that all expressions are invariant under $n \rightarrow-n$ [see Eq. (1.1)].

The second discussion point concerns the analytic $q^{2}$ continuation from spacelike to timelike values. Such a step is needed in evaluating the DY graphs with virtual gluons. Premature identification $n=-q$ in DI scattering no longer allows for an analytic continuation of the vertex function from spacelike to timelike $q^{2}$ values. Since $n^{2}$ may not change in this step, the vertex functions are evaluated for arbitrary $n$. After analytic $q^{2}$ continuation, $n$ is identified as indicated earlier.

The mass and infrared regularization procedures are our third discussion point. The (axial gauge) cut graphs contributing to the DI/DY processes are mass and/or infrared singular. They are regularized by on/off-shell mass assignments as defined and investigated, in the framework of the Feynman gauge, in Ref. 7. In our subsequent analysis we use the (undisputed) on-shell mass assignment and similarly determine the cross section for the (disputed) "conventional" off-shell mass assignment assuming both massive and massless quarks. In earlier analyses, the latter choice led to inconsistencies which could be resolved by the double-cut rule. 7,11 Our investigation therefore focuses partially on the results of a massive off-shell assignment which is in agreement with the above rule. We refer to it as the "correct off-shell" mass assignment. The reason for calculating the conventional off-shell assignment with massive as well as massless quarks is threefold: the importance of the quark rest mass becomes transparent. The (conventional) massless assignment permits the study of characteristic features which are also present 
in the correct off-shell assignment. The latter, however, suffers from the disadvantage of technically involved calculations and, in addition, leads to anomalous cuts. Finally, we gain more confidence in our findings. We define the mass assignments.

On-shell: All external momenta are on-shell $p_{i}{ }^{2}=m^{2}$. The gluon is given an infinitesimal rest mass $\lambda$. In Figs. 1 and 2 we otherwise set $m_{5}{ }^{2}$ $=\lambda^{2}, m_{6}{ }^{2}=q^{2}, m_{i}{ }^{2}=m^{2}$.

Conventional massive off-shell: All quarks have a nonvanishing rest mass $(m \neq 0)$. A chosen initial line (external) has in all cut graphs the same off-shell value ${p_{i}}^{2}=m^{2}-\mu^{2}$ with $\mu^{2}>0$. Finalstate mass regularization in DI scattering is constrained by unitarity (Cutkosky rules).

Conventional massless off-shell: We take over the preceding definition and introduce the changes: all quark rest masses vanish $(m=0)$ and ${p_{i}}^{2}=-\mu_{i}{ }^{2}$ with $\mu_{i}^{2}>0$.

Correct off-shell: The mass assignment is constrained by the double-cut rule with the masses in Figs. 1 and 2 fixed as $m_{1}{ }^{2}=m_{2}{ }^{2}=m_{8}{ }^{2}=m_{12}{ }^{2}$ $=m^{2}-\mu^{2}$ (with $\left.\mu^{2}>0\right), m_{5}{ }^{2}=0, m_{6}{ }^{2}=q^{2}, m_{i}{ }^{2}=m^{2}$ otherwise.

Note that the above mass assignments are symmetric with respect to the two quark lines. The correct off-shell mass assignment involves anomalous cuts with complex expressions in the intermediate steps. Since in the physical observables they all must cancel, we restrict ourselves to the real parts only. In Tables I-IX we therefore suppress all complex pieces which originate from the anomalous cuts. However, the vertex correction needs special care. While carrying out $q^{2}$ analytic continuation, the anomalous cuts must not be ignored and the imaginary parts can be disposed of only after this step has been completed. The conventional off-shell assignment takes all external lines in the initial state off-shell so that this problem is avoided. The M/IR singularities arising from the spurious $(n \cdot k)$ terms in Eq. (1.1) are also regularized by the above mass assignments. For on-shell, the $(n \cdot k)$ pole needs special care since $(n \cdot k)$ changes sign in the $k$ integration range and therefore vanishes. We elaborate on this point later on near $\mathrm{Eq}$. (A23). In the off-shell case, all terms proportional to $\left(p_{i}{ }^{2}-m_{i}{ }^{2}\right)^{k}$ in the numerator must be carefully evaluated since they often give rise to corresponding denominator terms and cancellation takes place. This remark particularly applies to the virtual-gluon (Appendix A) and the softgluon contributions (Appendix $\mathrm{C}$ ).

In this fourth discussion point we indicate how renormalization proceeds in the axial gauge. The ultraviolet (UV) singularities are removed by $n$ - dimensional regularization and mass-shell subtraction. Determination of the wave-function renormalization constants in the axial gauge is slightly more complicated than in a covariant gauge. Following Ref. 12, the (axial gauge) selfenergy can be decomposed as

$$
\begin{aligned}
\Sigma(p, n)= & Y_{1}+Y_{2} \not h+(\not p-m) X_{1} \\
& +h\left(p^{2}-m^{2}\right) X_{2}+\Sigma^{R}(p, n) .
\end{aligned}
$$

The self-mass is obtained by putting $\not p=m$ in Eq. (2.1),

$$
\delta m=Y_{1}+\left.\frac{p \cdot n}{m} Y_{2}\right|_{\phi=m}
$$

and the quark wave-function renormalization constant is given by

$$
\begin{aligned}
& 1-Z_{2}^{-1}=X_{1}+2(p \cdot n) X_{2}, \\
& X_{1}=\left.\frac{\partial \Sigma}{\partial \not p}\right|_{\phi=m}, \\
& X_{2}=\left.\frac{\partial \Sigma}{\partial p^{2}}\right|_{p^{2}=m^{2}} .
\end{aligned}
$$

$Y_{i}$ and $X_{i}$ depend on $p \cdot n, n^{2}$, and the mass parameters. The electromagnetic vertex correction in the axial gauge reads ${ }^{12}$

$$
\Gamma^{\mu}\left(p_{1}, p_{2}, n\right)=\gamma^{\mu} F+\gamma^{\mu} h p_{1} F^{\prime}+p_{2} h F^{\prime \prime} \text {. }
$$

The $F, F^{\prime}$, and $F^{\prime \prime}$ are functions of $q^{2}, p_{i} \cdot n$, and $n^{2}$. All UV divergence is confined to the first term $F$ in Eq. (2.4). In the actual calculation, $\Gamma^{\mu}$ is contracted as $\left(\not_{2}+m_{2}\right) \Gamma^{\mu}\left(\not_{1}+m_{1}\right)$. Therefore, in the Bjorken limit only the first term survives since all others lead to expressions proportional to $p_{i}{ }^{2}$. Note that for the off-shell assignment only the pole terms satisfy the Ward identity $Z_{1}=Z_{2}$; the on-shell assignment follows it exactly.

\section{DEEP-INELASTIC SCATTERING}

In this section we present our results on the DI structure function $\mathfrak{F}_{2}\left(z, q^{2}\right)$. By entering into the calculational details we aim at a discussion of the axial-gauge difficulties and their solution. Our presentation successively considers the individual terms and analyzes their dependence on the earlier defined mass assignments.

\section{A. Graphs and notation}

The order $-g^{2}$ DI cut graphs are shown in Fig. 1. Using the notation of Ref. 7(a), the parton structure functions are composed of

$$
\begin{aligned}
\mathcal{F}\left(z, q^{2}\right)= & \left(\mathcal{F}^{\mathrm{v} x}+\mathcal{F}^{S_{1}}+\mathcal{F}^{S_{2}}\right) \\
& +\left(\mathcal{F}^{R-\text { hard }}+\mathcal{F}^{R-\text { sof }}\right) .
\end{aligned}
$$




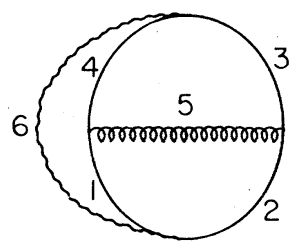

a

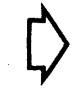
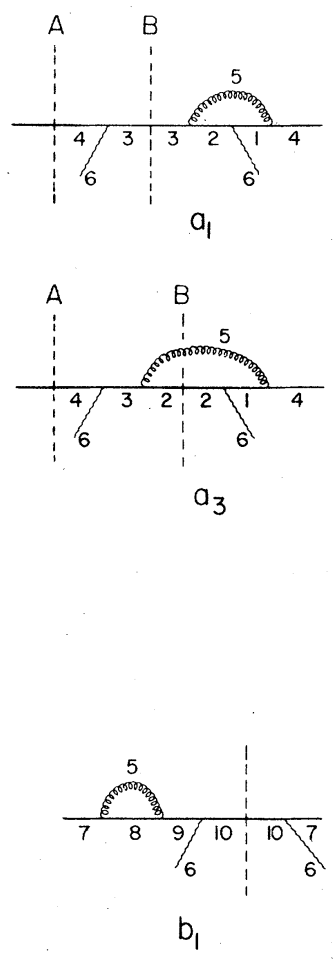

$10 \square$
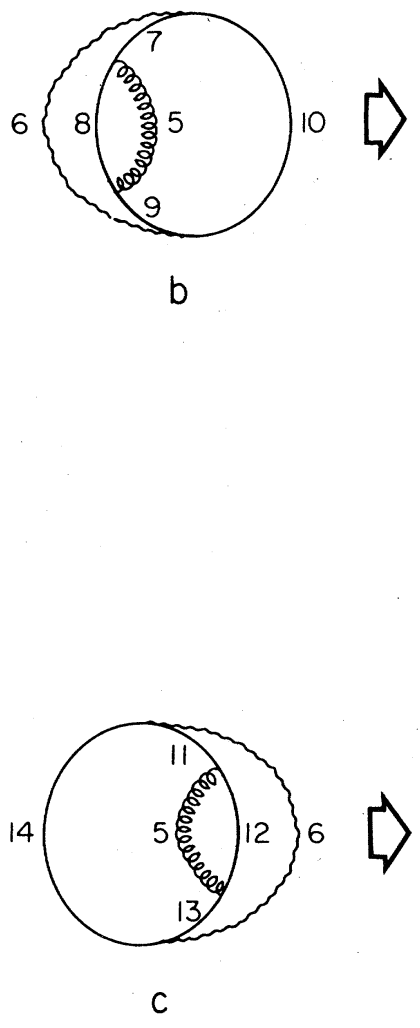

QUARK
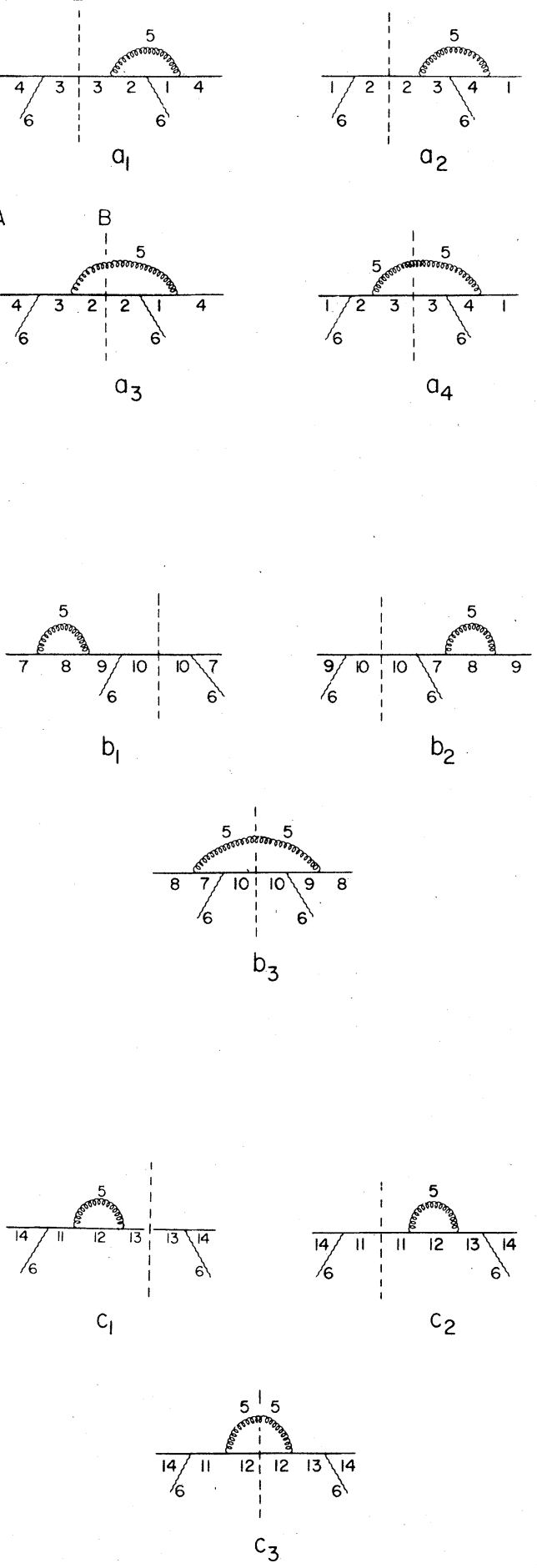

FIG. 1. Order $-g^{2}$ double-cut graphs of DI scattering on a quark target. Corresponding lines are indicated by numbers. 

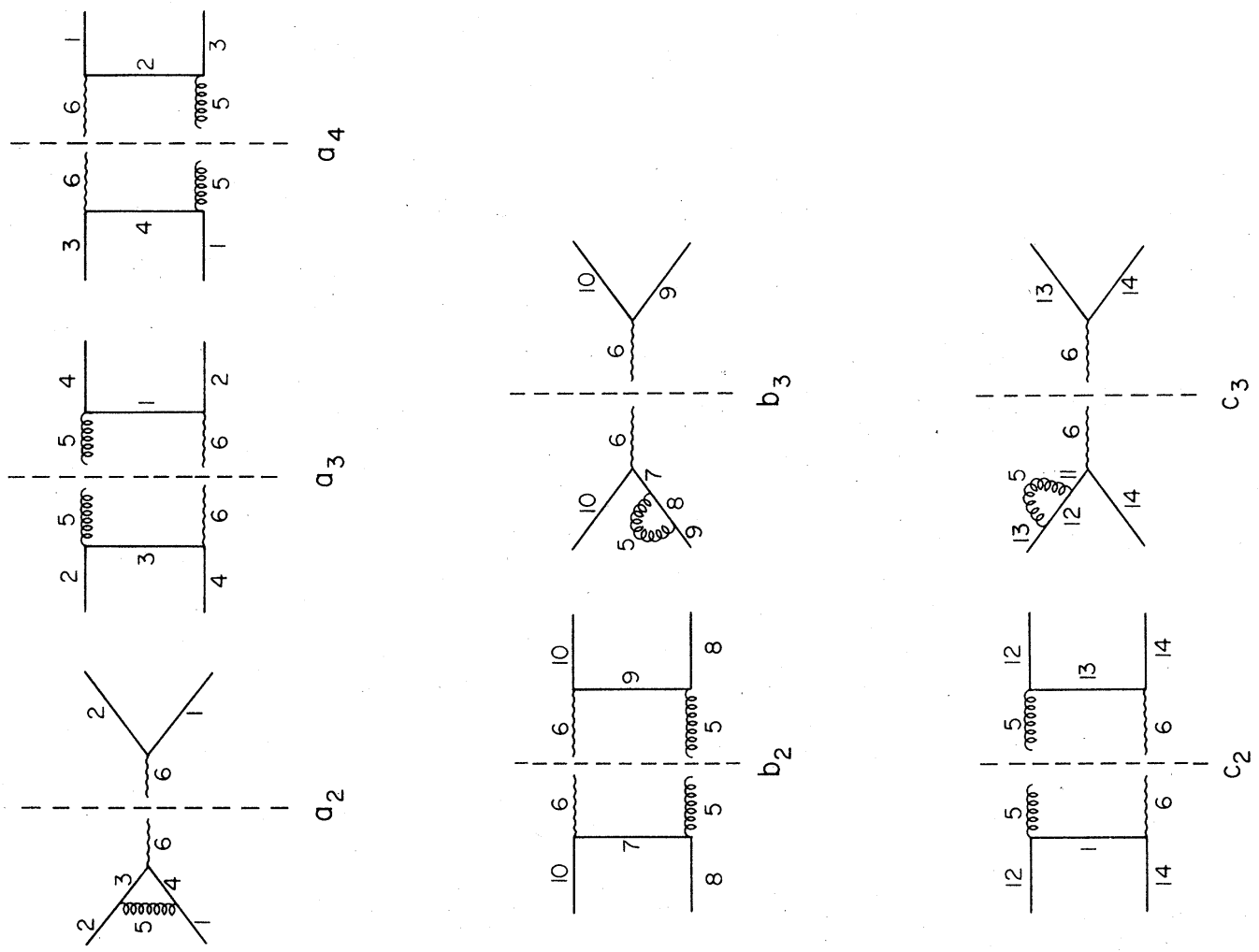

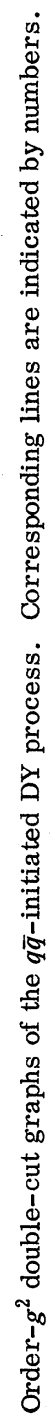
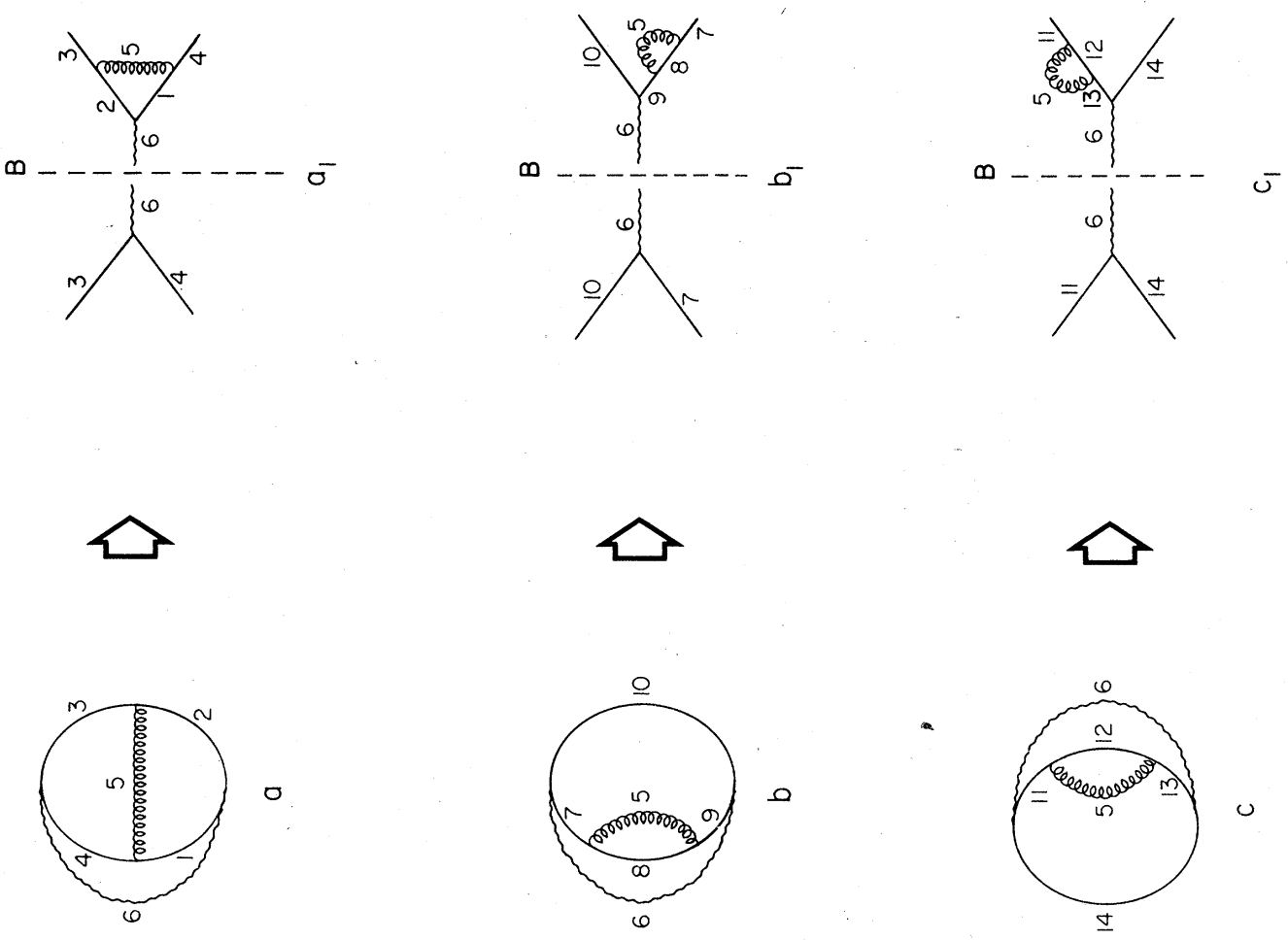

نे 
The superscript $V x$ refers to the unrenormalized electromagnetic vertex correction, whereas $S_{i}$ indicates the initial $\left(S_{1}\right)$ and the final $\left(S_{2}\right)$ state self-energies. $\mathcal{F}^{R^{-h} \text { hard }}$ is the hard-gluon structure function $(z \neq 1)$; it results from the real-gluon contribution $\mathcal{F}^{R}$ by taking the Bjorken limit. The softgluon contribution $(z \approx 1)$ defined by

$$
\mathcal{F}^{R-\text { sof t }}\left(q^{2}, \epsilon\right)=\delta(1-z) \int_{s_{0}}^{-\epsilon q^{2}} \frac{d s}{-q^{2}} \mathcal{F}^{R}\left(s, q^{2}, m_{i}{ }^{2}\right)
$$

contains the mass (and infrared) singularities which are prevented by the mass assignments. $s_{0}$ is the threshold value and $\epsilon$ stands for a momentum cutoff. For convenience we drop in the tables the terms proportional to $\ln ^{i} \epsilon$; they are easily obtained by reintegrating the hard-gluon part $\int_{0}^{1-\epsilon} d z \mathcal{F}^{R-h a r d}\left(z, q^{2}\right)$. Since in the axial gauge each contribution in Eq. (3.1) is built up by the three terms $(n \cdot k)^{-a}(a=0,1,2)$, they are all split up as

$$
\mathcal{F}=\mathcal{F}(0)+\mathcal{F}(1)+\mathcal{F}(2)
$$

corresponding to the three values of $a$.

\section{B. The individual contributions}

We consider the electromagnetic vertex correction of Figs. $1\left(a_{1}\right)$ and $1\left(a_{2}\right)$. For on-shell mass assignment and general $n$ the asymptotic forms are found:

$$
\begin{aligned}
& F^{\mathrm{Vx}}(0)=\frac{\alpha_{s}}{3 \pi}(-\xi+\ln \frac{\mathrm{M}^{2}}{-q^{2}}-\ln \frac{m^{2}}{-q^{2}}+2 \ln \frac{m^{2}}{\lambda^{2}} \ln \frac{m^{2}}{-q^{2}} \\
&\left.-4 \ln \frac{m^{2}}{-q^{2}}+\frac{\pi^{2}}{3}\right) \\
& F^{\mathrm{V}_{x}}(1)=\frac{\alpha_{s}}{3 \pi} \sum_{i=1}^{2}[+\xi-2+2 \ln \frac{m^{2}}{\mathrm{M}^{2}}-\ln \frac{4(p) n)^{2}}{-n^{2} \mathrm{M}^{2}} \\
&+\frac{1}{2} \ln ^{2} \frac{4\left(p_{i} \cdot n\right)^{2}}{-n^{2} m^{2}} \\
&\left.+\ln \frac{m^{2}}{\lambda^{2}} \ln \frac{4\left(p_{i} \cdot n\right)^{2}}{-n^{2} m^{2}}-\frac{\pi^{2}}{6}\right] \\
& F^{\mathrm{Vx}}(2)=\frac{\alpha_{s}}{3 \pi}\left(2 \xi+2 \ln \frac{\lambda^{2}}{\mathrm{M}^{2}}\right)
\end{aligned}
$$

where $\xi=2 /(n-4)-\gamma+\ln 4 \pi$. We point to the complete $M$ cancellation in their sum

$$
\begin{aligned}
F^{V x}=\frac{\alpha_{s}}{3 \pi}[ & 3 \xi-3 \ln \frac{\mathrm{M}^{2}}{-q^{2}}-\ln \frac{4\left(p_{1} \cdot n\right)^{2}}{-n^{2} \lambda^{2}} \\
& -\ln \frac{4\left(p_{2} \cdot n\right)^{2}}{-n^{2} \lambda^{2}}-\ln ^{2} \frac{-q^{2}}{\lambda^{2}} \\
& \left.+\frac{1}{2} \ln ^{2} \frac{4\left(p_{1} \cdot n\right)^{2}}{-n^{2} \lambda^{2}}+\frac{1}{2} \ln ^{2} \frac{4\left(p_{2} \cdot n\right)^{2}}{-n^{2} \lambda^{2}}-4\right]
\end{aligned}
$$

which is a characteristic feature of the axial gauge. The left singularities in the complete electromagnetic vertex correction are therefore of IR origin (see discussion in Sec. III 3C). Upon identification of $n=-q$, the $\left(\ln \lambda^{2}\right)^{i}$ terms in Eqs. (3.4) and (3.5) cancel; those of Eq. (3.6) persist (see Table I, column 3). All $\pi^{2}$ terms in Eqs. (3.4) and (3.5) disappear in the sum. The analogous off-shell results, evaluated for $n=-q$, are listed in Table I (where $\mu_{1}^{2} \gg \dot{\mu}_{2}^{2}$ ). The same features emerge. As in the on-shell case, all mass singularities and $\pi^{2}$ terms disappear in $F^{V_{x}}$. The persisting IR logarithm is traced back to $F^{v x}(2)$. The complete contribution of the electromagnetic vertex correction to the DI structure function reads

$$
\mathcal{F}^{V x}=2 \delta(1-z) F^{V x} .
$$

We carry out the analogous analysis of the selfenergy using $\Sigma^{\prime}=1-Z_{2}^{-1}$. For on-shell mass assignment and unspecified $n$ one finds

$$
\begin{aligned}
& \Sigma^{\prime}(0)=\frac{\alpha_{s}}{3 \pi}\left(\xi-\ln \frac{\mathrm{M}^{2}}{m^{2}}+2 \ln \frac{m^{2}}{\lambda^{2}}-4\right), \\
& \Sigma^{\prime}(1)=\frac{\alpha_{s}}{3 \pi}\left[-2 \xi-4 \ln \frac{m^{2}}{\mathrm{M}^{2}}+2 \ln \frac{4(p \cdot n)^{2}}{-n^{2} \mathrm{M}^{2}}-\ln ^{2} \frac{4(p \cdot n)^{2}}{-n^{2} m^{2}}\right. \\
& \left.-2 \ln \frac{m^{2}}{\lambda^{2}} \ln \frac{4(p \cdot n)^{2}}{-n^{2} m^{2}}+\frac{\pi^{2}}{3}+4\right] \\
& \Sigma^{\prime}(2)=\frac{\alpha_{s}}{3 \pi}\left(-2 \xi-2 \ln \frac{\lambda^{2}}{\mathrm{M}^{2}}\right) \text {. }
\end{aligned}
$$

The complete sum leaves

$$
\begin{aligned}
\Sigma^{\prime}=1-Z_{2}{ }^{-1}=\frac{\alpha_{s}}{3 \pi} & -3 \xi-3 \ln \frac{m^{2}}{\mathrm{M}^{2}}+2 \ln \frac{4(p \cdot n)^{2}}{-n^{2} \lambda^{2}} \\
& -\ln ^{2} \frac{4(p \cdot n)^{2}}{-n^{2} m^{2}} \\
& \left.-2 \ln \frac{m^{2}}{\lambda^{2}} \ln \frac{4(p \cdot n)^{2}}{-n^{2} m^{2}}+2 \ln \frac{m^{2}}{\lambda^{2}}+\frac{\pi^{2}}{3}\right] .
\end{aligned}
$$

From this result we learn that, in the axial gauge, $Z_{2}$ becomes $p$ dependent-a result which is distinct from the covariant gauge. Comparing the above results, Eqs. (3.9)-(3.11), with those for the vertex correction, Eqs. (3.4)-(3.6), we observe the relation

$$
\frac{1}{2}\left[\Sigma_{1}^{\prime}(a)+\Sigma_{2}^{\prime}(a)\right]=-F^{V x}(a) \quad(a=1,2) .
$$

The expression $F^{V} \equiv F^{V x}+\frac{1}{2}\left[\Sigma_{1}^{\prime}+\Sigma_{2}^{\prime}\right]$ therefore is gauge invariant and: consequently $n$ independent. All $\ln ^{2}$ and $\pi^{2}$ terms which disappear in $F^{V x}$ while going from the Feynman to the axial gauge now surface in $\Sigma^{\prime}$ [see Eq. (3.10)]. Both terms are traced back to the integral $I_{5}$ [see Eq. (A8)] which will be analyzed later. The analogous off-shell 

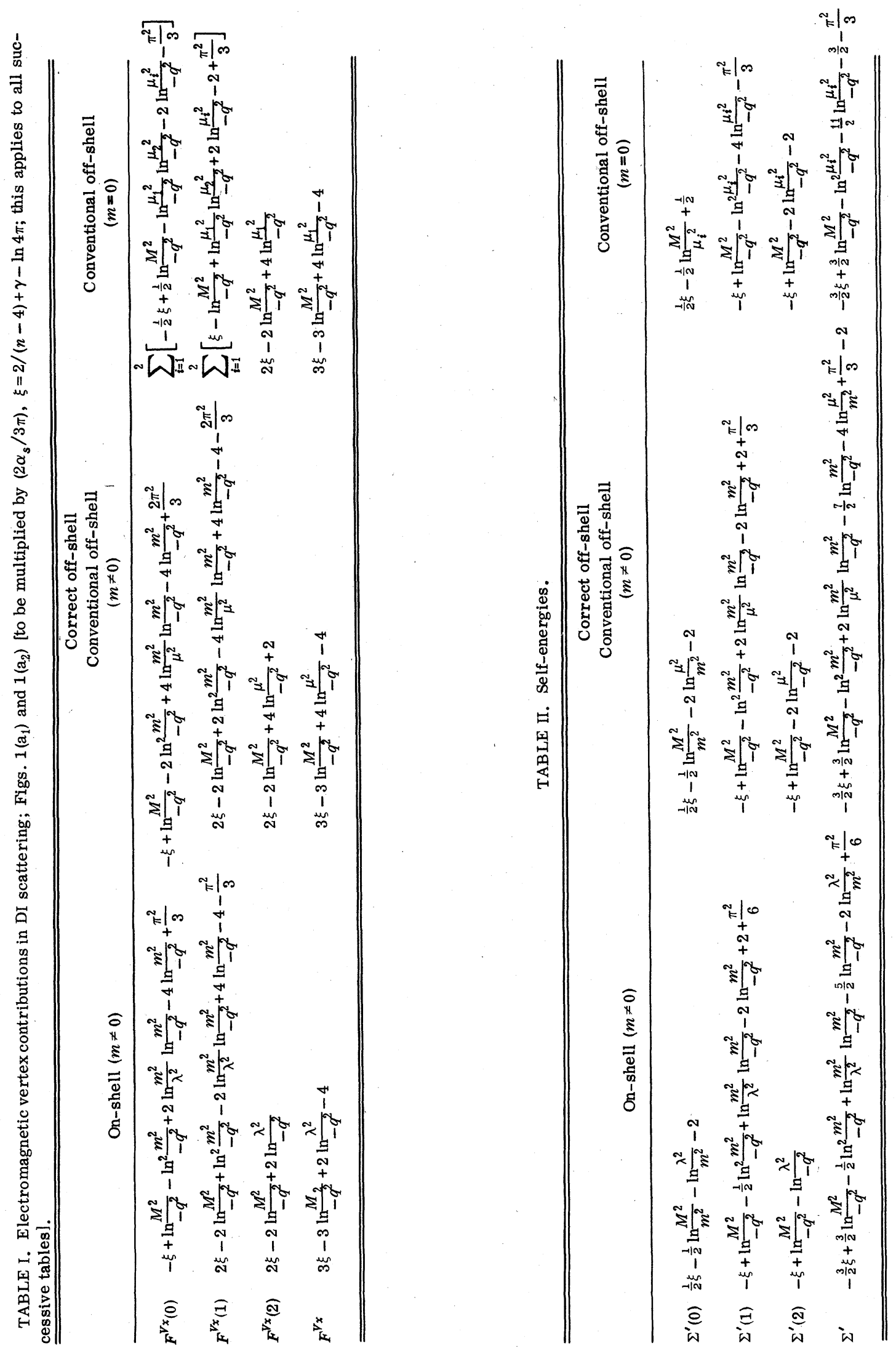
(and also on-shell) results evaluated for $n=-q$, are shown in Table II. The relation (3.13) now no longer holds true and a gauge-dependent renormalized vertex $F^{V}$ is found. All $\mathrm{In}^{2}$ and $\pi^{2}$ terms originate from $\Sigma^{\prime}(1)$ (as for on-shell), and both also appear with opposite signs in $F^{V x}(1)$ (see Table I). Comparing the $\ln ^{2}$ coefficient in $\Sigma^{\prime}$ for different mass assignments, we notice the appearance of a factor 2 while going from on-shell to off-shell. This phenomenon is also observed in $F^{V x}(0)$ and $F^{V x}(1)$ (Sudakov form factor). The contribution of the self-energy $S_{i}$ to the DI structure function takes the form

$$
\mathcal{F} s_{i}=\delta(1-z) \Sigma_{i}^{\prime} \text {. }
$$

The hard-gluon contributions from the outer rainbow, Fig. $1\left(b_{3}\right)$, the inner rainbow, Fig. $1\left(c_{3}\right)$, and the interference graphs, Figs. $1\left(a_{3}\right)$ and $\left(a_{4}\right)$, are evaluated by maintaining a completely general mass assignment which permits immediate specialization to the mass choices defined in Sec. III. Each line in these figures is given a number. For the mass assignments employed in this paper we can make a simplification in the momentum and mass notations; for this purpose the translation key will be indicated. Apart from $n^{2}<0, n^{\mu}$ is kept arbitrary, which results in lengthy expressions. The structure of the outer rainbow graph in Fig. $1\left(\mathbf{b}_{3}\right)\left(p_{8} \rightarrow p_{1}, m_{7}=m_{9} \rightarrow m_{x}, m_{5} \rightarrow \lambda\right)$ becomes transparent from

$$
\begin{aligned}
& \mathcal{F}^{R-\operatorname{hard}}(0)=\frac{2 \alpha_{s}}{3 \pi}\left[(1-z) \ln \left(\frac{-q^{2}}{z^{2} M_{1}^{2}}\right)+3 z-\frac{4\left(p_{1}^{2}\right)^{1 / 2} m_{x}-{p_{1}}^{2}-m_{x}^{2}-\lambda^{2}}{M_{1}^{2}}\right] \text {, } \\
& \mathcal{F}^{R-\operatorname{hard}}(1)=\frac{2 \alpha_{s}}{3 \pi}\left\{\frac{+2 z}{1-z} \ln \left(\frac{-q^{2}}{z^{2} M_{1}{ }^{2}}\right)+\frac{1+z}{1-z} \ln \left|\frac{4\left(n p_{1}\right)^{2} z^{2} M_{1}{ }^{2}}{n^{2} q^{2}{N_{1}}^{2}}\right|-n\left(p_{1}-q\right) A^{\mathrm{DI}}+2\left(p_{1}{ }^{2}-m_{1}{ }^{2}\right)\left[\frac{z}{(1-z)^{2}{N_{1}}^{2}}-\frac{1}{{M_{1}}^{2}}\right]\right\} \text {, } \\
& \mathcal{F}^{R-\mathrm{hard}}(2)=\frac{2 \alpha_{s}}{3 \pi}\left(n^{2} B^{\mathrm{DI}}-\frac{2}{1-z}\right) .
\end{aligned}
$$

The interference graphs in Fig. $1\left(\mathrm{a}_{3}\right)\left(p_{4} \rightarrow p_{1}, m_{1} \rightarrow m_{x}, m_{5} \rightarrow \lambda\right)$ and in Fig. $1\left(\mathrm{a}_{4}\right)\left(p_{1} \rightarrow p_{1}, m_{4} \rightarrow m_{x}, m_{5} \rightarrow \lambda\right)$ are analyzed in the same way leaving

$$
\begin{aligned}
& \mathcal{F}^{R-\text { hard }}(0)= \frac{2 \alpha_{s}}{3 \pi}\left[\frac{+2 z}{1-z} \ln \left(\frac{q^{2}}{z^{2} M_{1}^{2}}\right)-\frac{2 z}{1-z}\right], \\
& \mathcal{F}^{R-\mathrm{hard}}(1)=\frac{2 \alpha_{s}}{3 \pi}\left\{-\frac{2 z}{1-z} \ln \left(\frac{q^{2}}{z^{2} M_{1}^{2}}\right)-\frac{1+z}{1-z} \ln \left|\frac{4\left(n p_{1}\right)^{2} z^{2} M_{1}^{2}}{n^{2} q^{2} N_{1}^{2}}\right|\right. \\
&\left.+\left[\frac{n \cdot q(z-3)}{(1-z)}-\frac{2 z\left(p_{1} n\right)}{(1-z)}\right] A^{\mathrm{DI}}+\frac{1}{1-z}+\frac{n \cdot\left(p_{1}+q\right)}{p_{1} \cdot q(1-z)} C^{\mathrm{DI}}\right\}, \\
& \mathcal{F}^{R-\operatorname{hard}(2)=} \frac{2 \alpha_{s}}{3 \pi}\left(-2 n^{2} B^{\mathrm{DI}}+\frac{4}{1-z}\right) .
\end{aligned}
$$

For the inner rainbow graph Fig. 1 (c) diagram $c_{3}$ we get

$$
\begin{aligned}
& \mathcal{F}^{R-\text { hard }}(0)=\frac{2 \alpha_{s}}{3 \pi}\left(\frac{1}{2} \frac{1}{1-z}\right), \\
& \mathcal{F}^{R-\text { hard }}(1)=\frac{2 \alpha_{s}}{3 \pi}\left\{-\frac{1}{1-z}-\frac{n \cdot\left(p_{1}+q\right)}{p_{1} \cdot q(1-z)} C^{\mathrm{DI}}\right. \\
& \left.+\left[\frac{2 n \cdot q}{1-z}+\frac{n \cdot p_{1}(1+z)}{1-z}\right] A^{\mathrm{DI}}\right\}, \\
& \mathcal{F}^{R \text {-hard }}(2)=\frac{2 \alpha_{s}}{3 \pi}\left(n^{2} B^{\mathrm{DI}}-\frac{2}{1-z}\right) .
\end{aligned}
$$

The $A^{\mathrm{DI}}, B^{\mathrm{DI}}$, and $C^{\mathrm{DI}}$ in the preceding equations, defined by (B2), (B5), and (B6), are mass independent in the Bjorken limit and therefore remain mass regularization invariant. We have used a simplifying notation with

$$
\begin{gathered}
M_{i}^{2}=z^{-1}(1-z)^{-1}\left[-z(1-z) p_{i}^{2}\right. \\
\left.+(1-z) m_{x}^{2}+z \lambda^{2}\right], \\
N_{i}^{2}=(1-z)^{-1}\left(m_{x}^{2}-\lambda^{2}-z p_{i}^{2}\right)
\end{gathered}
$$

All hard-gluon parts remain, for the mass assignments of this paper, unaffected by $\lambda$. For $\lambda=0$ we find $M_{1}^{2}=z(1-z)^{-1} N_{1}^{2}$ which in Eqs. (3.16) and (3.19) leads to simplifications. The sum of all hard-gluon contributions reads 


$$
\begin{aligned}
& \mathcal{F}^{R-\text { hard }}=\frac{2 \alpha_{s}}{3 \pi}\left\{\frac{1+z^{2}}{1-z} \ln \left(\frac{-q^{2}}{z^{2} M_{1}^{2}}\right)+2\right. \\
& +3 z-\frac{3}{2} \frac{1}{1-z} \\
& -\frac{4 m_{x}\left(p_{1}^{2}\right)^{1 / 2}-p_{1}^{2}-m_{x}^{2}-\lambda^{2}}{M_{1}^{2}} \\
& \left.+2\left(p_{1}{ }^{2}-m_{1}{ }^{2}\right)\left[\frac{z}{(1-z)^{2}{N_{1}}^{2}}-\frac{1}{M_{1}^{2}}\right]\right\} \text {. }
\end{aligned}
$$

We observe that the complete hard-gluon structure function [Eq. (3.26)] is $n$ independent and $n$ can be given any value, e.g., $n= \pm q$. Further note that all initial-state mass singularities which appear in DI scattering can be attributed to the outer rainbow graph, Fig. 1(b) diagram $b_{3}$, and it becomes transparent if Eqs. (3.15) and (3.16) are added. The above expression, if calculated in the Feynman gauge, is unchanged apart from the typical off-shell term proportion to $\left(p_{1}{ }^{2}-m_{1}{ }^{2}\right)$. This term can be traced back to the $(1 / n \cdot k)$ part of the outer rainbow graph [Eq. (3.16)]. Using massive quarks, the off-shell term drops out in the Bjorken limit leading to a gauge-independent result for $\mathcal{F}^{R \text {-hard }}$. For massless off-shell assignment, however, it persists and contributes the extra term $-2 z /(1-z)$ to the structure function. In this case $\mathcal{F}^{R \text {-hard }}$ becomes gauge dependent. This gauge dependence is also observed if a covariant gauge different from the Feynman gauge is chosen. ${ }^{8}$

The soft-gluon contributions follow from diagram-by-diagram evaluation of the defining integral in Eq. (3.2). Our results are listed in Table III, Fig. $1\left(b_{3}\right)$, Table IV, Figs. $1\left(a_{3}\right)$ and $1\left(a_{4}\right)$, and Table V, Fig. $1\left(c_{3}\right)$. In Table IV we have taken $\mu_{1}^{2}$ $\gg \mu_{2}{ }^{2}$. As in the virtual-gluon corrections, the soft-gluon contributions are split into three parts corresponding to the $(n \cdot k)^{-a}(a=0,1,2)$ terms of the gluon propagator. Analyzing the individual graphs, we observe the following characteristics: $\mathcal{F}^{R \text {-soft }}(\mathrm{a})$ of the interference graphs, Figs. $1\left(\mathrm{a}_{3}\right)$ and $1\left(\mathrm{a}_{4}\right)$, Table IV, shows the same features as the corresponding vertex corrections $\mathfrak{F}^{V \times}(\mathrm{a})$, Figs. $1\left(a_{1}\right)$ and $1\left(a_{2}\right)$, Table I. In particular, the M singularities vanish and $\pi^{2}$ cancellation between the $a=0$ and $a=1$ components takes place. For $n=-q$, the leftover IR singularities originate from $\mathfrak{F}^{R \text {-soft }}(2)$. Furthermore, the M/IR singularities of the outer rainbow graph, Fig. $1\left(b_{3}\right)$, Table III, manifest themselves in $\mathcal{F}^{R \text {-soft }}(\mathrm{a})$ in the same way as observed in the corresponding external self-

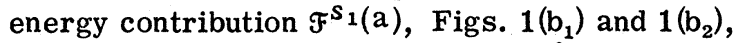
Table II. Notice the $\pi^{2}$ term in $\mathcal{F}^{R-\text { soft }}(1)$; it appears with an opposite sign in $\mathcal{F}^{S_{1}}$ (a). The same characteristics emerge from the inner rainbow

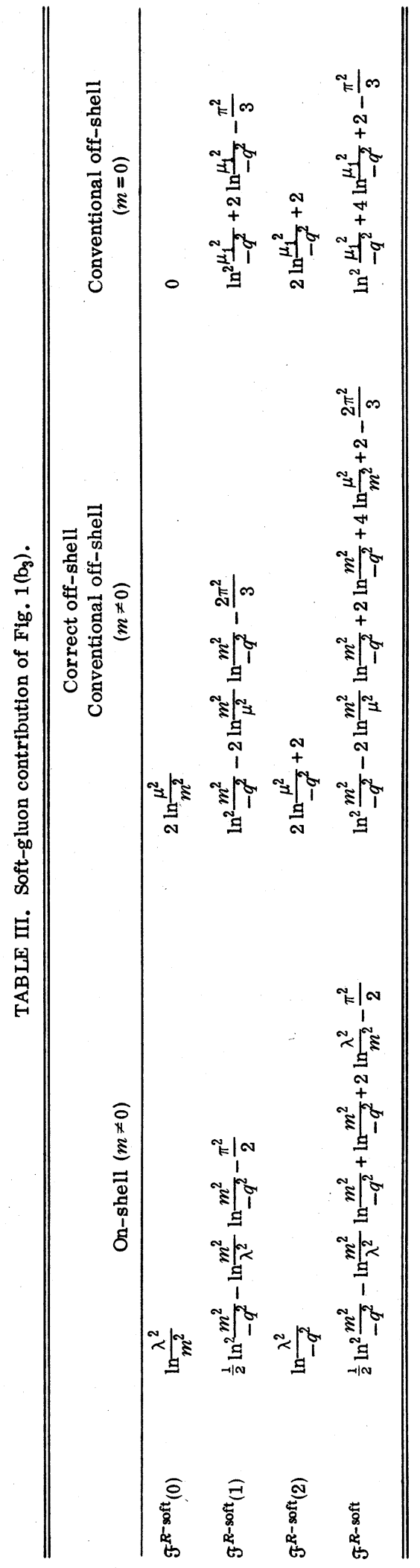


TABLE IV. Soft-gluon contribution of Figs. $1\left(a_{3}\right)$ and $1\left(a_{4}\right)$.

\begin{tabular}{|c|c|c|c|}
\hline & On-shell $(m=0)$ & $\begin{array}{l}\text { Correct off-shell } \\
\text { Conventional off-shell } \\
\qquad(m \neq 0)\end{array}$ & $\begin{array}{l}\text { Conventional off-shell } \\
\qquad(m=0)\end{array}$ \\
\hline $\mathfrak{F}^{R-\text { soft }}(0)$ & $\ln ^{2} \frac{m^{2}}{-q^{2}}-2 \ln \frac{m^{2}}{\lambda^{2}} \ln \frac{m^{2}}{-q^{2}}+2 \ln \frac{m^{2}}{-q^{2}}-\frac{2 \pi^{2}}{3}$ & $2 \ln ^{2} \frac{m^{2}}{-q^{2}}-4 \ln \frac{m^{2}}{\mu^{2}} \ln \frac{m^{2}}{-q^{2}}+2 \ln \frac{m^{2}}{-q^{2}}-\pi^{2}$ & $\sum_{i=1}^{2}\left[\ln \frac{\mu_{1}^{2}}{-q^{2}} \ln \frac{\mu_{2}^{2}}{-q^{2}}+\ln \frac{\mu_{i}^{2}}{-q^{2}}\right]$ \\
\hline $\mathfrak{F}^{R-\text { soft }}(1)$ & $-\ln ^{2} \frac{m^{2}}{-q^{2}}+2 \ln \frac{m^{2}}{\lambda^{2}} \ln \frac{m^{2}}{-q^{2}}-2 \ln \frac{m^{2}}{-q^{2}}+\frac{2 \pi^{2}}{3}$ & $-2 \ln ^{2} \frac{m}{-q^{2}}+4 \ln \frac{m^{2}}{\mu^{2}} \ln \frac{m^{2}}{-q^{2}}-2 \ln \frac{m^{2}}{-q^{2}}+\pi^{2}$ & $-\sum_{i=1}^{2}\left[\ln \frac{\mu_{1}^{2}}{-q^{2}} \ln \frac{\mu_{2}^{2}}{-q^{2}}+\ln \frac{\mu_{i}^{2}}{-q^{2}}\right.$ \\
\hline $\mathfrak{F}^{R-\text { soft }}(2)$ & $-2 \ln \frac{\lambda^{2}}{-q^{2}}$ & $-4 \ln \frac{\mu^{2}}{-q^{2}}-2$ & $-4 \ln \frac{\mu_{1}^{2}}{-q^{2}}$ \\
\hline $\mathfrak{F}^{R^{- \text {soft }}}$ & $-2 \ln \frac{\lambda^{2}}{-q^{2}}$ & $-4 \ln \frac{\mu^{2}}{-q^{2}}-2$ & $-4 \ln \frac{\mu_{1}^{2}}{-q^{2}}$ \\
\hline
\end{tabular}

graph, Fig. $1\left(c_{3}\right)$, Table V, and the final state self-energy contribution $\mathfrak{F}^{s_{2}}(\mathrm{a})$, Fig. $1\left(\mathrm{c}_{1}\right)$ and $1\left(c_{2}\right)$, Table II.

\section{DI structure functions in the axial gauge}

In Table VI we have assembled the complete DI structure function in the axial and also in the Feynman gauge. For on-shell and massive offshell assignments, both gauges lead to the same result. Choosing massless off-shell, however, reveals gauge dependence which is traced back to the outer rainbow graph, Fig. $1\left(b_{3}\right)$, and the external self-energies, Figs. $1\left(b_{1}\right)$ and $1\left(b_{2}\right)$. The $\pi^{2}$ terms, however, remain unaffected. Consider the M/IR cancellation between the individual cut graphs in Fig. 1. Due to the fact that in the axial gauge the gluon keeps its physical polarizations, M singularities only occur if in a Feynman graph two equal propagators appear. From this we infer that the $M$ singularities can only be attributed to the Compton graphs corresponding to Figs. $1\left(b_{1}\right)-1\left(b_{3}\right)$. Invoking unitarity, we therefore conclude that all $M$ and IR singularities are canceled in the following combinations: $\mathcal{F}^{R \text {-hard }}\left(\mathrm{a}_{3}\right)+\mathfrak{F}^{R-\text { soft }}\left(\mathrm{a}_{3}\right)+\mathcal{F}^{V x}\left(\mathrm{a}_{1}\right)$, $\mathfrak{F}^{R \text {-hard }}\left(\mathrm{a}_{4}\right)+\mathfrak{F}^{R \text {-soft }}\left(\mathrm{a}_{4}\right)+\mathfrak{F}^{V x}\left(\mathrm{a}_{2}\right)$, and $\mathfrak{F}^{R \text {-hard }}\left(\mathrm{c}_{3}\right)$ $+\mathcal{F}^{R \text {-soft }}\left(\mathrm{c}_{3}\right)+\mathcal{F}^{S_{2}}\left(\mathrm{c}_{1}+\mathrm{c}_{2}\right)$. One further observes that the above combinations are independent of the particular M/IR regularizations since we have respected the unitarity relation between Compton and cut graphs for all $\mathrm{M}$ assignments (including the "conventional"). The unitarity argument does not apply for the DY process. We will find later that the combination involving interf erence and vertex graphs is M/IR regularization independent only if a correct mass assignment is chosen. From the above we conclude that all $\mathrm{M}$ singularities, and with it all $M$ regularization dependence, can be attributed to the sum $\mathcal{F}^{R \text {-hard }}\left(\mathrm{b}_{3}\right)+\mathfrak{F}^{R \text {-soft }}\left(\mathrm{b}_{3}\right)+\mathcal{F}^{S_{1}}\left(\left(\mathrm{~b}_{1}\right)\right.$ $+\left(b_{2}\right)$ ). In order to achieve proper IR cancellation in the above combination of structure functions (in particular for massless off-shell), $Z_{2}$ must be evaluated at the external (internal) mass pole. This requirement follows from the fact that $\mathcal{F}^{R \text {-soft }}\left(\mathrm{b}_{3}\right)\left[\mathfrak{F}^{R \text {-soft }}\left(\mathrm{c}_{3}\right)\right]$ was calculated with a specific external (internal mass). ${ }^{3}$ (This also applies to the Feynman gauge, but here the mistake only shows up in the constant terms.) If $Z_{2}$ is not evaluated in the way indicated above, the next-to-leading IR logs will not compensate, leaving uncanceled IR singularities in the DI structure function (the above rule was not followed in Refs. 5 and 6 ).

\section{DRELL-YAN PROCESS}

In this section we analogously analyze the DY parton cross section. The order $-g^{2}$ DY cut graphs, shown in Fig. 2, lead to the contributions

$$
\begin{aligned}
W\left(\tau, Q^{2}\right)= & \left(W^{V x}+W^{S_{1}}+W^{S_{2}}\right) \\
& +\left(W^{R \text {-hard }}+W^{R-\text { soft }}\right) .
\end{aligned}
$$

Each of them is composed of the three terms in the axial propagator with $a=0,1,2$. Our notation follows closely that of DI scattering. Apart from presenting our results, we focus in this section on those aspects which are different and/or less transparent in DI scattering.

\section{A. The individual contributions}

The electromagnetic vertex corrections, Figs. $2\left(a_{1}\right)$ and $2\left(a_{2}\right)$, are obtained from those in DI scattering via analytic continuation from spacelike to timelike $q^{2}$ values; one sets $p_{1} \rightarrow p_{1}, p_{2} \rightarrow-p_{2}$ with $n$ fixed. Subsequently, one may identify $n^{2}=-Q^{2}$. Our results are listed in Table VII (with the imaginary parts being dropped). For on-shell mass assignment [see Eqs. (3.4)-(3.6)], only $W^{V x}(0)$ gets an extra $\pi^{2}$ term [ $W^{V x}(1)$ is $q^{2}$ independent]. For conventional off-shell, the analytic continuation, leads to an extra $2 \pi^{2}$ term in $W^{V x}(0)$ (Sudakov form). For correct off-shell, however, the net 
effect is zero. The latter result is due to the presence of anomalous cuts in Figs. $1\left(a_{1}\right)$ and $2\left(a_{1}\right)$. While performing the analytic continuation, these cuts must be taken into account. The off-shell regularization, contrary to on-shell, also leads to extra $\pi^{2}$ terms in $W^{V x}(1)$. They originate from the typical off-shell terms $\left(p_{i}^{2}-m_{i}^{2}\right)^{k}$. The $\pi^{2}$ term is provided by the integral $I$ defined in Eq. (A7). The same feature is observed for massive off-shell in $W^{V x}(2)$. Here, the $\pi^{2}$ is given by the integral $J$ defined in Eq. (A17). The self-energies appearing in Figs. $2\left(b_{1}\right), 2\left(b_{3}\right), 2\left(c_{1}\right)$, and $2\left(c_{3}\right)$ can be taken over from those obtained in DI scattering (Table II). There is no analytic continuation involved.

We focus on the graphs describing real gluon production shown in Figs. $2\left(a_{3}\right), 2\left(a_{4}\right), 2\left(b_{2}\right)$, and $2\left(c_{2}\right)$ and evaluate their hard-gluon parts. As we did earlier in DI scattering, we keep the most general mass assignment possible. The translation key is again indicated in brackets in order to facilitate an easy correspondence between the notation of the specific double-cut graph and the general one which applies to all figures. The outer rainbows, Fig. $2\left(\mathrm{~b}_{2}\right),\left(p_{8} \rightarrow p_{2}, p_{10} \rightarrow p_{1}, m_{7}=m_{9}-m_{x}, m_{5}\right.$ $-\lambda)$ and Fig. 2(c $\left.c_{2}\right)\left(p_{12}-p_{1}, p_{14}-p_{2}, m_{11}=m_{13} \rightarrow m_{x}\right.$, $\left.m_{5}-\lambda\right)$, both lead to the same result,

$$
\begin{aligned}
W^{R-h a r d}(0)=\frac{2 \alpha_{s}}{3 \pi}\left[(1-\tau) \ln \frac{Q^{2}(1-\tau)}{\tau^{2} M_{i}^{2}}-(1-\tau)\right. \\
\left.-\frac{4 m_{x}\left(p_{i}{ }^{2}\right)^{1 / 2}-p_{i}{ }^{2}-m_{x}{ }^{2}-\lambda^{2}}{\bar{M}_{i}^{2}}\right],
\end{aligned}
$$

$$
\begin{aligned}
W^{R-h a r d}(1)=\frac{2 \alpha_{s}}{3 \pi}\{ & \frac{2 \tau}{1-\tau} \ln \frac{Q^{2}(1-\tau)}{\tau^{2} M_{i}^{2}} \\
& +\frac{1+\tau}{1-\tau} \ln \left|\frac{4\left(n \cdot p_{i}\right)^{2} \tau^{2} M_{i}^{2}}{n^{2} Q^{2}(1-\tau) N_{i}^{2}}\right| \\
& +n \cdot\left(p_{1}+p_{2}\right) A^{\mathrm{DY}} \\
& \left.+2\left(p_{i}^{2}-m_{i}^{2}\right)\left[\frac{\tau}{(1-\tau)^{2} N_{i}^{2}}-\frac{1}{M_{i}^{2}}\right]\right\}
\end{aligned}
$$

$W^{R-h a r d}(2)=\frac{2 \alpha_{s}}{3 \pi}\left(\frac{-2}{1-\tau}\right)$

Each of the interference graphs shown in Fig. $2\left(\mathrm{a}_{3}\right)\left(p_{2} \rightarrow p_{1}, p_{4} \rightarrow p_{2}, m_{1}=m_{3} \rightarrow m_{x}, m_{5}-\lambda\right)$ and in Fig. 2( $\left(\mathrm{a}_{4}\right)\left(p_{1} \rightarrow p_{2}, p_{3} \rightarrow p_{1}, m_{2}=m_{4} \rightarrow m_{x}, m_{5} \rightarrow \lambda\right)$ lead to the same structure 


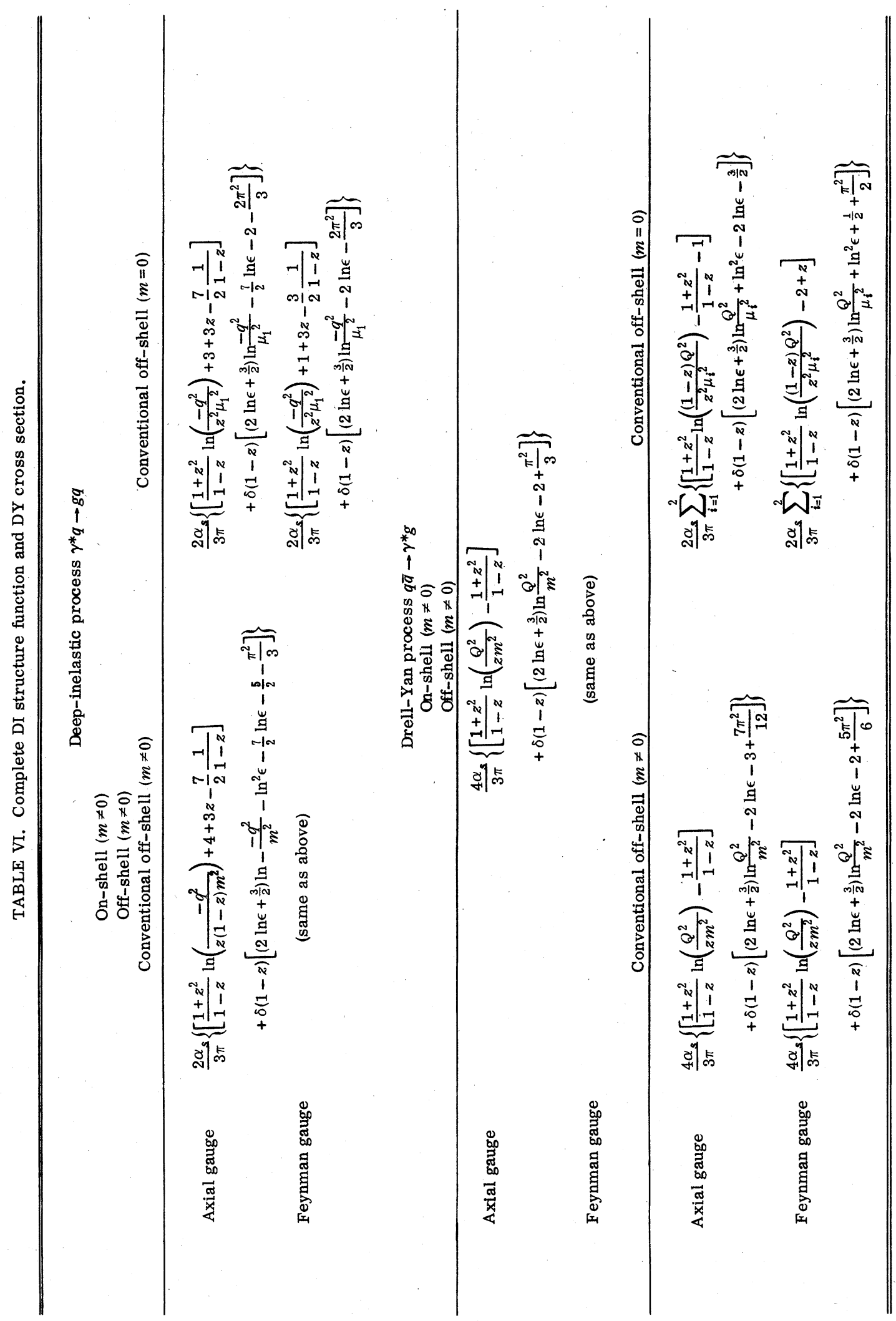




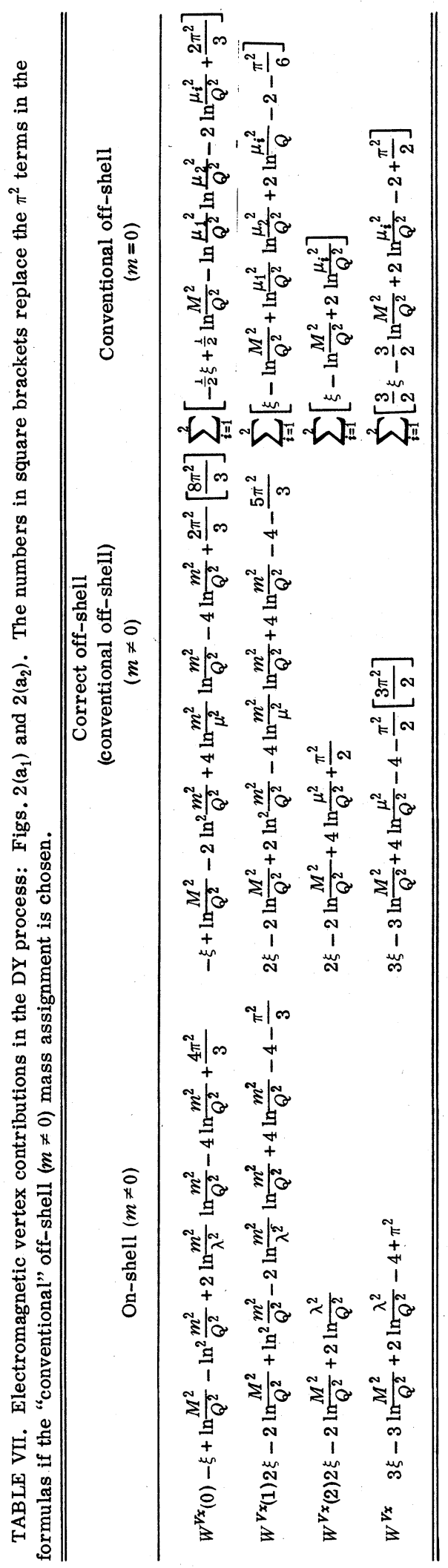

$$
\begin{aligned}
& W^{R-h a r d}(0)=\frac{2 \alpha_{s}}{3 \pi} \sum_{i=1}^{2}\left[\frac{\tau}{1-\tau} \ln \frac{Q^{2}(1-\tau)}{\tau^{2} M_{i}^{2}}\right] \\
& W^{R-h a r d}(1)=\frac{2 \alpha_{s}}{3 \pi} \sum_{i=1}^{2}\left[\frac{-\tau}{1-\tau} \ln \frac{Q^{2}(1-\tau)}{\tau^{2} M_{i}^{2}}\right. \\
& -\frac{1}{2} \frac{1+\tau}{1-\tau} \ln \left|\frac{4\left(n \cdot p_{i}\right)^{2} \tau^{2} M_{i}^{2}}{n^{2} Q^{2}(1-\tau) N_{i}^{2}}\right| \\
& \left.-\frac{1}{2} n \cdot\left(p_{1}+p_{2}\right) A^{\mathrm{DY}}\right] \text {, } \\
& W^{R-h a r d}(2)=\frac{2 \alpha_{s}}{3 \pi}\left(\frac{2}{1-\tau}\right) .
\end{aligned}
$$

$M_{i}$ and $N_{i}$ are defined in Eqs. (3.24) and (3.25) and for the mass-singularity-free $A^{D Y}$ we refer to Eq. (B9). The properties pointed out while discussing the analogous results for DI scattering apply for the above results, Eqs. (4.2)-(4.7), in the same way. We point in particular to the offshell term of Eq. (4.3) which only contributes for massless off-shell and which is $n$ independent. The complete DY hard-gluon part

$$
\begin{aligned}
W^{R-h a r d}=\frac{2 \alpha_{s}}{3 \pi} \sum_{i=1}^{2}\{ & \frac{1+\tau^{2}}{1-\tau} \ln \frac{Q^{2}(1-\tau)}{\tau^{2} M_{i}^{2}}-(1-\tau) \\
& -\frac{4 m_{x}\left(p_{i}^{2}\right)^{1 / 2}-p_{i}^{2}-m_{x}^{2}-\lambda^{2}}{M_{i}^{2}} \\
& \left.+2\left(p_{i}^{2}-m_{i}^{2}\right)\left[\frac{\tau}{(1-\tau)^{2} N_{i}^{2}}-\frac{1}{M_{i}^{2}}\right]\right\}
\end{aligned}
$$

is also $n$ independent. This insight justifies our procedure to choose in DI scattering $n$ different from its value in the DY process. Note that for massive quarks, $W^{R \text {-hard }}$ is gauge-independent. As in DI scattering, all initial-state $M$ singularities originate from the ladder graphs, Figs. $2\left(\mathrm{~b}_{2}\right)$ and $2\left(c_{2}\right)$, whereas the interference graphs, Figs. 2( $\left(a_{3}\right)$ and $2\left(\mathrm{a}_{4}\right)$, are $M$ finite.

With the preceding results, we are able to determine the soft-gluon integrals which are defined as in Eq. (3.2). Our results for the outer rainbows shown in Figs. $2\left(b_{2}\right)$ and $2\left(c_{2}\right)$ are listed in Table VIII. The M/IR singularities manifest themselves in much the same way as in the analogous DI graphs, Fig. $1\left(b_{3}\right)$, Table III. The results of the interference graphs in Figs. $2\left(a_{3}\right)$ and $2\left(a_{4}\right)$ are assembled in Table IX. The M/IR characteristics of the components $W^{R-s o f t}(a=0,1,2)$ are the same as in the analogous DI graphs, Figs. $1\left(a_{3}\right)$ and $1\left(a_{4}\right)$, Table IV. In particular, cancellation of the $\mathrm{M} /$ IR singularities takes place if $W^{R-s o f t}(0)$ and $W^{R-\text { soft }}(1)$ are added. In the complete sum of 
TABLE VIII. Soft-gluon contribution of Figs. $2\left(\mathrm{~b}_{2}\right)$ and $2\left(\mathrm{c}_{2}\right)$.

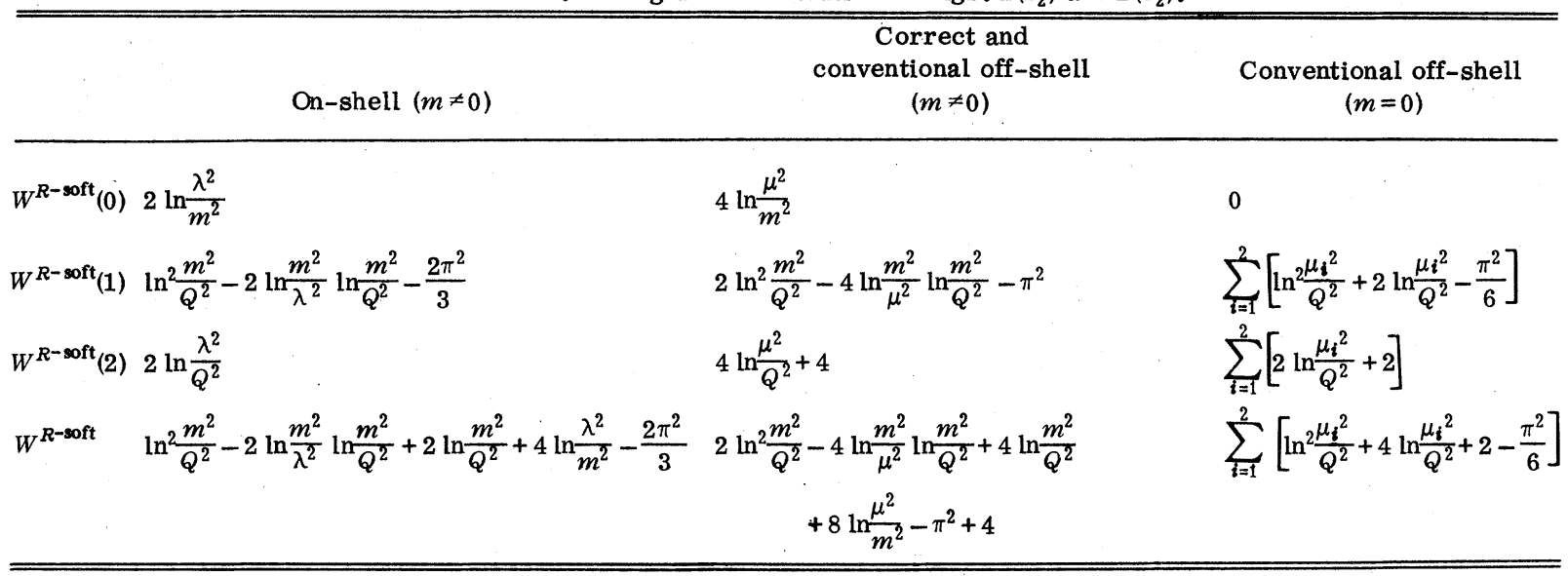

$W^{R-s o f t}$, only a single IR logarithm is left which can be traced back to $W^{R-s o f t}(2)$. For on-shell and for conventional off-shell, the $\pi^{2}$ terms of $W^{R-\text { soft }}(0)$ and $W^{R-s o f t}(1)$ compensate with rational numbers only (and no $\pi^{2}$ terms) left over. For correct off-shell $W^{R-s o f t}(1)$ and $W^{R-s o f t}(2)$ contribute $\pi^{2}$ terms which, however, will remain. They originate from $\bar{I}$ [see Eq. (C4)] and from $\bar{J}$ [see Eq. (C7)]. These integrals are associated with the offshell terms $\left(p_{i}{ }^{2}-m_{i}{ }^{2}\right)^{k}$ which, in the asymptotic limit, do not vanish. The absence of any $\pi^{2}$ terms for conventional off-shell is a direct consequence of the different mass choices. We elaborate on this point later on.

\section{B. Drell-Yan cross section in the axial gauge}

The complete expression for the DY cross section is given in Table VI. Comparing the Feynmangauge result with the axial-gauge result, we observe, for the hard-gluon part $(z \neq 1)$, the same features as in DI scattering. This does not apply, however, for the $\delta(1-z)$ contribution to $W$. While going from the Feynman to the axial gauge we notice, for conventional off-shell, a change in the $\pi^{2}$ terms. If the correct massive off-shell and the conventional massive off-shell are compared, a difference in the $\delta(1-z)$ term becomes transparent. It appears in both gauges. The correct massive off-shell leads to the same result as found for on-shell.

The cancellation of the M/IR singularities proceeds in the same way as discussed in DI scattering (Sec. III C). We form the combination of cut diagrams which belong to the same bubble graph in Fig. 2, and observe that, similar to DI scattering, the sum of vertex corrections, Figs. 2( $\left.a_{1}\right)$ and $2\left(a_{2}\right)$, and interference graphs, Figs. 2( $\left.a_{3}\right)$ and $2\left(a_{4}\right)$ is finite in the axial gauge. However, the sum $W^{R-h a r d}\left(\left(a_{3}\right)+\left(a_{4}\right)\right)+W^{R-s o f t}\left(\left(a_{3}\right)+\left(a_{4}\right)\right)$ $+W^{V x}\left(\left(a_{1}\right)+\left(a_{2}\right)\right)$ is $M / I R$ regularization independent only if a cons istent mass assignment is chosen (see remark in Sec. IIIC). In the axial gauge, all $M$ singularities can be attributed to Figs. $2\left(b_{1}\right)-2\left(b_{3}\right)$, $2\left(c_{1}\right)-2\left(c_{3}\right)$. The combination $W^{R-h a r d}\left(b_{2}\right)+W^{R-s o f t}$ $+W^{R-s o f t}\left(\mathrm{~b}_{2}\right)+W^{S_{1}}\left(\left(\mathrm{~b}_{1}\right)+\left(\mathrm{b}_{3}\right)\right)$ is therefore $M$ regular-

TABLE IX. Soft-gluon contributions of Figs. $2\left(a_{3}\right)$ and $2\left(a_{4}\right)$. The numbers in square brackets replace the $\pi^{2}$ terms in the formulas if the "conventional" off-shell $(m \neq 0)$ mass assignment is chosen.

\begin{tabular}{|c|c|c|c|}
\hline & On shell $(m \neq 0)$ & $\begin{array}{c}\text { Correct off shell } \\
\text { [conventional off shell] } \\
\qquad(m \neq 0)\end{array}$ & $\begin{array}{l}\text { Conventional off-shell } \\
\qquad(m=0)\end{array}$ \\
\hline$W^{R-\text { soft }}(0)$ & $\ln ^{2} \frac{m^{2}}{Q^{2}}-2 \ln \frac{m^{2}}{\lambda^{2}} \ln \frac{m^{2}}{Q^{2}}-\frac{2 \pi^{2}}{3}$ & $2 \ln ^{2} \frac{m^{2}}{Q^{2}}-4 \ln \frac{m^{2}}{\mu^{2}} \ln \frac{m^{2}}{Q^{2}}\left[-\pi^{2}\right]$ & {$\left[\frac{\mu_{1}^{2}}{Q^{2}} \ln \frac{\mu_{2}^{2}}{Q^{2}}-\frac{\pi^{2}}{6}\right]$} \\
\hline$W^{R-\mathrm{soft}_{(1)}}$ & $-\ln ^{2} \frac{m^{2}}{Q^{2}}+2 \ln \frac{m^{2}}{\lambda^{2}} \ln \frac{m^{2}}{Q^{2}}+\frac{2 \pi^{2}}{3}$ & $-2 \ln ^{2} \frac{m^{2}}{Q^{2}}+4 \ln \frac{m^{2}}{\mu^{2}} \ln \frac{m^{2}}{Q^{2}}+2 \pi^{2}\left[+\pi^{2}\right]$ & $\left.-\ln \frac{\mu_{1}^{2}}{Q^{2}} \ln \frac{\mu_{2}^{2}}{Q^{2}}+\frac{\pi^{2}}{6}\right]$ \\
\hline$W^{R-\text { soft }}(2)$ & $-2 \ln \frac{\lambda^{2}}{Q^{2}}$ & $-4 \ln \frac{\mu^{2}}{Q^{2}}-\frac{\pi^{2}}{2}[-2]$ & $\sum_{i=1}^{2}\left[-2 \ln \frac{\mu_{i}^{2}}{Q^{2}}\right]$ \\
\hline$W^{R-\text { soft }}$ & $-2 \ln \frac{\lambda^{2}}{Q^{2}}$ & $-4 \ln \frac{\mu^{2}}{Q^{2}}+\frac{3 \pi^{2}}{2}[-2]$ & $\sum_{i=1}^{2}\left[-2 \ln \frac{\mu_{i}^{2}}{Q^{2}}\right]$ \\
\hline
\end{tabular}


ization dependent. The same applies for the sum of contributions coming from Figs. $2\left(c_{1}\right)-2\left(c_{3}\right)$.

We here expand on the cancellation of the IR singularities. That their complete cancellation sensitively depends on a consistent M/IR regularization scheme was already discussed in Ref. 7 .
We demonstrate the subtle points by a (simplified but illustrative) example. Using the eikonal approximation of Ref. 11, IR compensation between the virtual and real (soft) gluon contributions is exposed. In the vertex correction Fig. $2\left(a_{2}\right)$ integrals of the form

$$
\begin{gathered}
V=i \int \frac{d^{4} k}{(2 \pi)^{4}} \frac{p_{1 \lambda} p_{2 \sigma} p^{\lambda \sigma}}{\left[k^{2}+i \epsilon\right]\left[\left(k-p_{1}\right)^{2}-m_{3}{ }^{2}+i \epsilon\right]\left[\left(k+p_{2}\right)^{2}-m_{4}{ }^{2}+i \epsilon\right]} \\
\underset{\text { eikonal approximation }}{\approx} \frac{d^{4} k}{(2 \pi)^{3}} \delta^{+}\left(k^{2}\right) \frac{p_{1 \lambda} p_{2 \sigma} p^{\lambda \sigma}}{\left[-2 k \cdot p_{1}+m_{1}{ }^{2}-m_{3}{ }^{2}+i \epsilon\right]\left[2 k \cdot p_{2}+m_{2}{ }^{2}-m_{4}{ }^{2}+i \epsilon\right]}
\end{gathered}
$$

appear (see Appendix A). $P^{\lambda \sigma}$ stands for the spin part of the gluon propagator in any gauge and, in particular, in the axial gauge [Eq. (1.1)]. The corresponding soft-gluon contribution, Fig. $2\left(a_{4}\right)$, reads

$$
S=\int \frac{d^{4} k}{(2 \pi)^{3}} \delta^{+}\left(k^{2}\right) \frac{p_{1 \lambda} p_{2 \sigma} \mathrm{P}^{\lambda \sigma}}{\left[-2 k \cdot p_{1}+m_{1}^{\prime 2}-m_{4}^{\prime 2}+i \epsilon\right]\left[-2 k \cdot p_{2}+m_{3}^{\prime 2}-m_{2}^{\prime 2}+i \epsilon\right]} .
$$

Note the masses $m_{i}, m_{i}^{\prime}$ are a priori kept general. IR cancellation between $V$ and $S$ will only take place if

$$
\begin{aligned}
& {m_{1}}^{2}-{m_{3}}^{2}={m_{1}^{\prime 2}}^{2}-{m_{4}^{\prime}}^{2} \\
& {m_{2}}^{2}-{m_{4}}^{2}=m_{2}^{\prime 2}-m_{3}^{\prime 2} .
\end{aligned}
$$

The on-shell mass assignment satisfies Eq. (4.12) trivially. The conventional off-shell mass assignment (with $m_{1}^{2}=m_{1}^{\prime 2}=m^{2}-\mu_{1}^{2}, m_{2}^{2}=m_{3}^{\prime 2}=m^{2}-\mu_{2}^{2}$, $\left.m_{3}{ }^{2}=m_{4}^{2}=m_{2}^{\prime 2}=m_{4}^{\prime 2}=m^{2}\right)$ does not satisfy this condition and the sum $(V+S)$ is therefore IR regularization and gauge dependent. In the correct off-shell mass assignment (defined by $m_{1}{ }^{2}=m_{1}^{\prime 2}=m^{2}-\mu_{1}^{2}$, $m_{2}^{2}=m_{2}^{\prime 2}=m^{2}-\mu_{2}^{2}, m_{3}^{2}=m_{3}^{\prime 2}=m_{4}^{2}=m_{4}^{\prime 2}=m^{2}$ ), however, uniform IR cancellation takes place leaving $(V+S)$ IR regularization and gauge independent. Examples of $V$ and $S$ type integrals of the typical off-shell term $\left(p_{i}{ }^{2}-m_{i}{ }^{2}\right)^{k}$ are shown in Appendices A and C. We focus our interest on the integrals $I,(\mathrm{~A} 7)$, and $J,(\mathrm{~A} 17)$, which we already consider in connection with the DY vertex correction. For correct off-shell, their $\pi^{2}$ terms are cancelled by the corresponding soft integral $\bar{I}$, (C4), and $\bar{J},(\mathrm{C} 7)$, since $I=\bar{I}$ and $J=\bar{J}$. For conventional off-shell, these latter relations still hold true as long as $q^{2}<0$. For $q^{2}>0$, however, the equalities turn to inequalities and the $\pi^{2}$ compensation no longer takes place.

\section{DRELL-YAN CORRECTION TERM}

The above analysis of the order $-g^{2}$ parton cross sections and, in particular, the emphas is on their nonlogarithm contributions, is motivated by mass factorization. The $M / I R$ regularization-free determination of the nonleading contributions in the $\mathrm{RG}$ approach is sought. In the lowest order the
DY correction term is given by

$$
\Delta_{q \bar{Q}}=W\left(z, Q^{2}\right)-\mathcal{F}_{q}\left(z, Q^{2}\right)-\mathcal{F}_{\bar{q}}\left(z, Q^{2}\right) .
$$

The hard-gluon part of $\Delta_{\bar{q}}(z \neq 1)$ is M/IR regularization independent and it agrees with the Feynman-gauge calculation. However, the $\delta(1-z)$ part, as already seen in this latter gauge ${ }^{7}$ depends quite sensitively on the chosen mass assignment. Provided a consistent mass assignment (on-shell ${ }^{7}, 13$ correct off-shell, ${ }^{7} n$ dimensional $\left.{ }^{7}, 14\right)$ is chosen, one finds in the axial gauge (as well as in any other covariant gauge) the result

$$
\Delta_{a \bar{Q}}=\frac{2 \alpha_{s}}{3 \pi}\left(1+\frac{4 \pi^{2}}{3}\right)(\delta \text { part }) .
$$

However, conventional off-shell (with massive or massless quarks) provides a gauge-dependent result. In the covariant gauges one finds $\Delta_{a 8}$ $=\left(2 \alpha_{s} / 3 \pi\right)\left(1+7 \pi^{2} / 3\right)$ (Refs. $\left.8,10,15\right)$ (massive or massless), whereas in the axial gauge the second factor is changed to $\left(-1+11 \pi^{2} / 6\right)$ (massive quarks) or $\left(1+4 \pi^{2} / 3\right)$ (massless quarks). The agreement between this last result and Eq. (5.2) must be considered as accidental since $\Delta_{q \bar{q}}$ depends, for conventional off-shell, on the mass assignment and is gauge dependent.

One encounters the same problem if the total cross section for $e^{*} e^{-} \rightarrow \gamma^{*} \rightarrow q \bar{q} g$ is calculated. The conventional off-shell assignment violates unitarity between the vacuum polarization and the corresponding cut graphs,,$^{7,16}$ providing us with a result which is different from the one obtained by a consistent mass assignment which respects unitarity. This $M / I R$ regularization technique therefore provides us with nonunique and gauge-dependent cross section which is unacceptable.

We comment here on àn earlier study ${ }^{9}$ which ad- 
dresses itself to the mass regularization problem. The authors suggest that the use of the Zwanziger formalism ${ }^{7}$ still allows us to proceed with the conventional off-shell mass assignment. Their arguments are limited to the DY cross section and they defend the view that a continuous limit from onshell to conventional off-shell is possible. All order- $g^{2}$ DY parton cross sections reveal $\pi^{2}$ factors whose origin stems on one hand from the analytic continuation of the form factor and on the other hand from other Feynman graph contributions. The discussion in Ref. 9 is limited to the first type. Our study reveals, however, that there are additional $\pi^{2}$ 's whose coefficients are massassignment dependent. Since in the earlier studies they were ignored, we do not think that any firm conclusions are possible concerning their technique.

\section{SUMMARY AND CONCLUSIONS}

Using the principal-value definition for the spurious singularities in the axial gauge, we have calculated, in order $g^{2}$, the DI structure function for $\gamma^{*} q \rightarrow g q$ and the DY cross section $q \bar{q} \rightarrow \gamma^{*} g$. The highly sensitive nonlogarithm contributions, ignored in the previous work, were given special attention. If consistent $M / I R$ regularization methods are applied, the axial-gauge results agree. with the analogous analysis in a covariant gauge, and for all massive regularization schemes there is one and the same answer. In particular, the correct off-shell mass assignment leads to a gauge-independent (axial, covariant, etc., gauges) DY correction term and its form agrees with the analogous evaluations where undisputed IR/M techniques (on-shell, $n$ dimensional, etc.) were used. Our calculations do not confirm the $\pi^{2}$ term which originates from the analytic continuation of the electromagnetic vertex function in the case of the conventional off-shell mass assignment as found in Ref. 9. Notice, however, that in the latter reference, this term was obtained for $n^{2}>0$ whereas we used $n^{2}<0$. The conventional mass regularization applied in a covariant gauge provides us with a unique answer irrespective of whether massive or massless quarks are used. In the axial gauge, however, there is no longer agreement. We thus conclude that conventional off-shell leads to $M / I R$ regularization and gauge-dependent answers. Apart from clearing these (seemingly technical) points, our study gives insight into the complexity of the calculations in a general axial gauge. Analyses on this level have so far not been completed and the ways to overcome these technical problems have not been shown. With the present paper we also aim to fill this gap.

\section{APPENDIX A}

In this section we present some useful integrals which appear while calculating the virtual (vertex and self-energy) gluon contributions. Since the integrals containing the first term in the gluon propagator (Feynman gauge) are already given in the literature (see, e.g., Ref. 7b), we limit ourselves to those expressions in which the $(n \cdot k)^{-a}$ term appears:

$$
I_{1}=\int \frac{d^{m} k}{(2 \pi)^{m}} \frac{k}{(n \cdot k) D_{k} D_{i}}=A p_{i}+B n
$$

with

$$
D_{i}=\left(p_{i}-k\right)^{2}-m_{i}^{2}, \quad D_{k}=k^{2}-\lambda^{2},
$$$$
A=\frac{1}{G} \int \frac{d^{m} k}{(2 \pi)^{m}}\left[\frac{\left(n \cdot p_{i}\right)}{D_{i} D_{k}}-\frac{n^{2}}{2(n \cdot k) D_{i}}+\frac{n^{2}}{2(n \cdot k) D_{k}}\right.
$$$$
\left.-\frac{n^{2}\left(p i^{2}-m i^{2}\right)}{2(n \cdot k) D_{i} D_{k}}\right] \text {, }
$$

$$
\begin{aligned}
& B=\frac{1}{G} \int \frac{d^{m} k}{(2 \pi)^{m}}\left[\frac{\left(n \cdot p_{i}\right)}{2(n \cdot k) D_{i}}-\frac{\left(n \cdot p_{i}\right)}{2(n k) D_{k}}\right. \\
& \left.+\frac{p_{i}^{2}-m_{i}^{2}}{2(n \cdot k) D_{i} D_{k}}-\frac{p_{i}^{2}}{D_{i} D_{k}}\right] \\
& \underset{m i \rightarrow 0}{\approx} \frac{i}{(4 \pi)^{m / 2}} \frac{2\left(p_{i} \cdot n\right)}{n^{2}}\left[\Gamma\left(2-\frac{m}{2}\right)+2-\ln \frac{4\left(p_{i} \cdot n\right)^{2}}{-n^{2}}\right]
\end{aligned}
$$

$I_{4}=\int \frac{d^{m} k}{(2 \pi)^{m}} \frac{1}{(n \cdot k) k^{2} D_{1} D_{2}}=\frac{i}{(4 \pi)} \frac{m}{2} I$,

$$
\begin{aligned}
I=\int_{0}^{1} d x & \frac{1}{x\left(p_{1}{ }^{2}-m_{1}{ }^{2}\right)+(1-x)\left(p_{2}{ }^{2}-m_{2}{ }^{2}\right)} \frac{1}{\sqrt{N}} \\
& \times \ln \left(\frac{P \cdot n+\sqrt{N}}{-P \cdot n+\sqrt{N}}\right)
\end{aligned}
$$

where 


$$
\begin{aligned}
N & \equiv(P \cdot n)^{2}+n^{2}\left[x(1-x) q^{2}-x m_{1}^{2}-(1-x) m_{2}^{2}\right], \quad P=x p_{1}+(1-x) p_{2}, \quad q=p_{2}-p_{1} ; \\
I_{5} & =\int \frac{d^{m} k}{(2 \pi)^{m}} \frac{1}{(n \cdot k) D_{i} D_{k} \lambda, m_{i} \rightarrow 0}=\frac{i}{(4 \pi)^{m / 2}} \frac{1}{n \cdot p_{i}}\left[\frac{1}{4} \ln \frac{4\left(p_{i} \cdot n\right)^{2}}{-n^{2} m_{i}{ }^{2}}+\frac{1}{2} \ln \frac{m^{2}}{\lambda^{2}} \ln \frac{4\left(p_{i} \cdot n\right)^{2}}{-n^{2} m_{i}{ }^{2}}-\frac{\pi^{2}}{12}\right] \text { (on-shell) } \\
& \left.=\frac{i}{(4 \pi)^{m / 2}} \frac{1}{n \cdot p_{i}}\left[\frac{1}{2} \ln { }^{2} \frac{4\left(p_{i} \cdot n\right)^{2}}{-n^{2} m_{i}{ }^{2}}+\ln \frac{m^{2}}{\mu^{2}} \ln \frac{4\left(p_{i} \cdot n\right)^{2}}{-n^{2} m_{i}{ }^{2}}-\frac{\pi^{2}}{6}\right] \quad \text { (off-shell } m_{i} \neq 0\right) \\
& =\frac{i}{(4 \pi)^{m / 2}} \frac{1}{n \cdot p_{i}}\left[\frac{1}{2} \ln \frac{4\left(p_{i} \cdot n\right)^{2}}{-n^{2} p_{i}{ }^{2}}+\frac{\pi^{2}}{6}\right] \quad\left(\text { off-shell } m_{i}=0\right) .
\end{aligned}
$$

The integrals with $\mathrm{a}(n \cdot k)^{2}$ in the denominator can be determined via $k$ differentiation. One finds

$$
\begin{aligned}
& I_{6}=\int \frac{d^{m} k}{(2 \pi)^{m}} \frac{1}{(n \cdot k)^{2} D_{k}}=\frac{i}{(4 \pi)^{m / 2}} \frac{2}{n^{2}}\left[\Gamma\left(2-\frac{m}{2}\right)+\ln \lambda^{2}\right], \\
& I_{7}=\int \frac{d^{m} k}{(2 \pi)^{m}} \frac{1}{(n \cdot k)^{2} D_{1} D_{2}}=\frac{-i}{(4 \pi)^{m / 2}} \int_{0}^{1} d x\left[\frac{P \cdot n}{N^{3 / 2}} \ln \left(\frac{P \cdot n+\sqrt{N}}{-P \cdot n+\sqrt{N}}\right)-\frac{2}{\sqrt{N}}\right],
\end{aligned}
$$

where $P$ and $N$ are defined near Eq. (A7),

$$
\begin{aligned}
& I_{8}=\int \frac{d^{m} k}{(2 \pi)^{m}} \frac{1}{(n \cdot k)^{2} D_{i}}=\frac{-i}{(4 \pi)^{m / 2}} \frac{2}{n^{2}}\left[\Gamma\left(2-\frac{m}{2}\right)-\ln \frac{4\left(p_{i} \cdot n\right)^{2}}{-n^{2}}\right], \\
& I_{9}=\int \frac{d^{m} k}{(2 \pi)^{m}} \frac{1}{(n \cdot k)^{2} D_{i} D_{k} p_{i}{ }^{2} \rightarrow m_{i}^{2}}=\frac{i}{(4 \pi)^{m / 2}} \frac{2}{n^{2}\left(p_{i}^{2}-m_{i}{ }^{2}\right)}\left[-\ln \frac{4\left(n \cdot p_{i}\right)^{2}}{-n^{2} \lambda^{2}}+2 \ln \frac{4\left(p_{i} \cdot n\right)^{2}}{n^{2}\left(p_{i}{ }^{2}-m_{i}{ }^{2}\right)}\right], \\
& I_{10}=\int \frac{d^{m} k}{(2 \pi)^{m}} \frac{1}{(n \cdot k)^{2} k^{2} D_{1} D_{2}}=\frac{1}{{p_{1}}^{2}-m_{1}{ }^{2}+{p_{2}}^{2}-m_{2}{ }^{2}}\left[2 I_{11}+I_{8}\left(p_{1}\right)+I_{8}\left(p_{2}\right)-2 I_{7}\right],
\end{aligned}
$$

where

$$
\begin{aligned}
& I_{11} \equiv-\left(p_{1}+p_{2}\right)_{\mu} \frac{\partial}{\partial n_{\mu}} I_{4}=\frac{i}{(4 \pi)^{m / 2}} J \\
& J \equiv \int_{0}^{1} d x \frac{1}{x\left(p_{1}^{2}-m_{1}{ }^{2}\right)+(1-x)\left(p_{2}{ }^{2}-m_{2}{ }^{2}\right)}\left[\frac{\left(p_{1}+p_{2}\right) \cdot P(P \cdot n)-n \cdot\left(p_{1}+p_{2}\right) P^{2}}{N^{3 / 2}} \ln \left(\frac{P \cdot n+\sqrt{N}}{-P \cdot n+\sqrt{N}}\right)\right. \\
& \left.+2 \frac{\left[(p \cdot n) n \cdot\left(p_{1}+p_{2}\right)-n^{2} P \cdot\left(p_{1}+p_{2}\right)\right]}{n^{2} N}\right] \\
& I_{12}=\int \frac{d^{m} k}{(2 \pi)^{m}} \frac{\not k}{(n \cdot k)^{2} D_{i} D_{k}}=C p_{i}+D \not h
\end{aligned}
$$

where $C$ and $D$ are given by

$$
\begin{gathered}
C=\frac{1}{G}\left[\left(n \cdot p_{i}\right) I_{5}-\frac{n^{2}}{2} I_{8}\right. \\
\left.+\frac{n^{2}}{2} I_{6}-\frac{n^{2}}{2}\left(p_{i}{ }^{2}-m_{i}{ }^{2}\right) I_{9}\right], \\
D=\frac{1}{G}\left[\frac{1}{2}\left(p_{i} \cdot n\right) I_{8}-\frac{1}{2}\left(n p_{i}\right) I_{6}\right. \\
\left.\quad+\frac{1}{2} p_{i} \cdot n\left(p_{i}{ }^{2}-m_{i}{ }^{2}\right) I_{9}-p_{i}{ }^{2} I_{5}\right]
\end{gathered}
$$

with $G$ defined near Eq. (A3).

We have tested unitarity on the (axial gauge) self-energy. The usual Feynman integral is first evaluated with the results listed in Eq. (3.12). Subsequently, we write a dispersion relation and fix the discontinuity via the Cutkosky rules. If unitarity holds, the two results must agree. We illustrate some on-shell subtleties on the integral $I_{5}$ [see (A8)]. Its discontinuity, evaluated via the Cutkosky rules, reads

$$
\begin{aligned}
\operatorname{Im} I_{5} & =\frac{1}{2(2 \pi)^{2}} \int d^{4} k \delta^{+}\left(k^{2}-\lambda^{2}\right) \delta^{+}\left((k-p)^{2}-m^{2}\right) \frac{1}{n \cdot k} \\
& =\frac{1}{16 \pi} \frac{1}{N} \ln \left|\frac{\mathrm{P} p_{0}+\mathrm{N}|\overrightarrow{\mathrm{p}}|}{\mathrm{P} p_{0}-\mathrm{N}|\overrightarrow{\mathrm{p}}|}\right|,
\end{aligned}
$$

where

$$
\begin{array}{r}
\mathrm{P} \equiv(n \cdot p), \quad \mathrm{N} \equiv\left[(n \cdot p)^{2}-n^{2} s\right]^{1 / 2}, \quad p_{0}=\frac{s+\lambda^{2}-m^{2}}{2 \sqrt{s}} \\
\left(s \equiv p^{2}\right) .
\end{array}
$$

Note $(1 / n \cdot k)$ in Eq. (A21) is always understood as a principal value as defined in Eq. (1.2). The $(n \cdot k)$ term changes sign while passing in the integration range $(m+\lambda)^{2}<s<\infty$ the point

$$
s_{p} \equiv m^{2}+\lambda^{2}+2 m \lambda\left(1-\frac{(p \cdot n)^{2}}{n^{2} m^{2}}\right)^{1 / 2}
$$

It follows from solving the equation which results if the denominator in the logarithm argument vanishes. Without the prescription of Eq. (1.2), 
$(n \cdot k)$ would give rise to a cut, since in the logarithm of Eq. (A21) the absolute sign would be absent. The dispersion integral

$$
I_{5}=\frac{1}{\pi} \int_{(m+\lambda)^{2}}^{\infty} d s \frac{\operatorname{Im} I_{5}}{s-m^{2}}
$$

is evaluated by using the discontinuity of Eq. (A21) and by separating the integration range into the two parts $\left[(m+\lambda)^{2}, s_{p}\right]$ and $\left[s_{p}, \infty\right]$. The result of this simple calculation agrees with the Feynman integral in Eq. (A8). For off-shell mass assignment there is no such pseudothreshold $s_{p}$ and the dispersion integral can be evaluated without additional difficulties.

\section{APPENDIX B}

In this section we present the phase-space integrals which appear in the hard-gluon part of the DI and DY processes. In DI scattering $\gamma^{*} q \rightarrow g q$ the phase-space integrals are generally denoted by

$$
I^{(i, j)}=\int d^{4} p_{2} d^{4} k \delta^{+}\left(p_{2}{ }^{2}-m_{2}{ }^{2}\right) \delta^{+}\left(k^{2}-\lambda^{2}\right) \delta^{4}\left(p_{1}+q-p_{2}-k\right) \frac{1}{(n \cdot k)^{2}\left[\left(p_{1}-k\right)^{2}-m_{x}{ }^{2}\right]^{j}} .
$$

We shall present here only the results which involve the $(n \cdot k)^{l}$ denominators. In shorthand notation they are given by

$$
\begin{aligned}
I^{(1,0)} & =\frac{\pi}{2} A^{\mathrm{DI}}=\frac{\pi}{2}\left(\frac{1}{b} \ln \frac{b+a}{b-a}\right) \\
I^{(1,1)} & =-\frac{\pi}{2} \frac{1}{\left(n \cdot p_{1}\right) s} \ln \left|\frac{4\left(p_{1} \cdot n\right)^{2} s}{n^{2} N_{1}^{4}}\right|, \\
I^{(2,0)} & =\frac{2 \pi}{n^{2}} \frac{1}{s} \\
I^{(2,-1)} & =-\pi B^{D I} \\
& \equiv-\pi\left(\frac{a c-s d}{b^{3 / 2}} \ln \frac{b+a}{b-a}+2 \frac{a d-n^{2} c}{n^{2} b^{2}}\right),
\end{aligned}
$$

$$
\begin{aligned}
& I^{(1,-1)}=-\pi C^{\mathrm{DI}} \\
& \equiv-\frac{\pi}{2}\left(s \frac{a d-n^{2} c}{b^{3 / 2}} \ln \frac{b+a}{b-a}+2 \frac{a c-s d}{b^{2}}\right), \\
& I^{(1,2)}=\frac{\pi}{2} \frac{1}{\left(n \cdot p_{1}\right) s} \frac{s-q^{2}}{N_{1}^{4}}, \\
& a=n(p+q), \quad b=\left(a^{2}-s n^{2}\right)^{1 / 2}, \\
& c=p_{1} \cdot\left(p_{1}+q\right), \quad d=\left(n \cdot p_{1}\right), \quad s=\left(p_{1}+q\right)^{2}, \\
& N_{i}{ }^{4}=q^{2} p_{i}{ }^{2}+\left(s-q^{2}\right)\left(m_{x}{ }^{2}-\lambda^{2}\right) \text {. }
\end{aligned}
$$

where

The phase-space integrals appearing in the DY process are generally denoted by

$$
J^{(\mathfrak{l}, j)}=\int d^{4} q d^{4} k \delta^{+}\left(q^{2}-Q^{2}\right) \delta^{+}\left(k^{2}-\lambda^{2}\right) \delta^{4}\left(p_{1}+p_{2}-q-k\right) \frac{1}{(n \cdot k)^{l}\left[\left(p_{i}-k\right)^{2}-m_{x}{ }^{2}\right]^{j}} .
$$

Their explicit evaluation reveals

$$
\begin{aligned}
& J^{(1,0)}=\frac{\pi}{2} A^{\mathrm{DY}}=\frac{\pi}{2} \frac{1}{f} \ln \frac{f+e}{f-e}, \\
& J^{(1,1)}=-\frac{\pi}{2} \frac{1}{\left(n \cdot p_{i}\right)\left(s-Q^{2}\right)} \ln \left|\frac{4\left(p_{i} \cdot n\right)^{2}\left(s-Q^{2}\right)}{n^{2} \bar{N}_{i}^{4}}\right|, \\
& J^{(2,0)}=\frac{2 \pi}{n^{2}} \frac{1}{s-Q^{2}}, \\
& J^{(1,2)}=\frac{\pi}{2} \frac{1}{\left(n \cdot p_{i}\right)\left(s-Q^{2}\right)} \frac{s}{\bar{N}_{i}{ }^{4}}, \\
& e=n \cdot\left(p_{1}+p_{2}\right), \quad f=\left(e^{2}-n^{2} s\right)^{1 / 2}, \\
& \bar{N}_{i}^{4}=s\left(m_{x}{ }^{2}-\lambda^{2}\right)-Q^{2} p_{i}{ }^{2} .
\end{aligned}
$$

APPENDIX C

In this appendix we present only those two softgluon integrals which lead to $\pi^{2}$ terms in the off- shell mass assignment and which contain the $(n \cdot k)^{l}$ denominator. For convenience purposes we choose here a calculation technique which differs from the one given in Eq. (3.2). We take advantage of the connection [see Ref. $7(\mathrm{~b})$ and Eq. (3.15)]

$$
W^{R-\text { soft }}=W^{R-\operatorname{conv}}(\omega)+\mathrm{L}(\omega, \epsilon),
$$

where $W^{R-\operatorname{conv}}(\omega)$ is simply the convective part of the diagrams describing real gluon production. If the numerator has no terms which contain the gluon momentum, we are allowed to put

$$
\omega=\frac{1}{2} \in \sqrt{Q^{2}} \Rightarrow \omega^{2} \gg m_{i}^{2}
$$

so that considerable simplifications occur since $L(\omega, \epsilon) \sim 0$. We thus may limit ourselves to the evaluation of

$$
\begin{array}{r}
B_{l} \equiv \int_{0}^{\omega} \frac{d^{3} k}{2 k} \frac{1}{(n \cdot k)^{l}} \frac{1}{\left[\left(p_{1}-k\right)^{2}-m_{1}^{2}\right]\left[\left(p_{2}-k\right)^{2}-m_{2}^{2}\right]} \\
(l=1,2),(\mathrm{C} 3)
\end{array}
$$


where $\omega$ is given in (C2). Straightforward calculation reveals

$$
B_{1} \underset{Q^{2} \gg m_{i}^{2}}{=} \frac{\pi}{2} \bar{I} \equiv \frac{\pi}{2} I\left(p_{1} \rightarrow p_{1}, p_{2} \rightarrow-p_{2}\right)
$$

with $I$ given in Eq. (A7). The $l=2$ integral reads

$$
B_{2}=\frac{1}{{p_{1}}^{2}-m_{1}{ }^{2}+p_{2}{ }^{2}-m_{2}^{2}}\left[2 R-T\left(p_{1}\right)+T\left(p_{2}\right)\right],
$$

where

$$
T\left(p_{i}\right) \equiv \int_{0}^{\infty} \frac{d^{3} k}{2 k} \frac{1}{(n \cdot k)^{2}} \frac{1}{\left[\left(k-p_{i}\right)^{2}-m_{i}^{2}\right]}
$$

is the soft analog of $I_{8}$ in Eq. (A13) providing a logarithm only. After the constraint (C2) has been imposed, one finds

$$
R_{Q^{2} \gg m_{i}^{2}}^{=} \frac{\pi}{2} \bar{J}=\frac{\pi}{2} J\left(p_{1} \rightarrow p_{1}, p_{2} \rightarrow-p_{2}\right)
$$

with $J$ defined in (A17). $\bar{I}$, (C4), and $\bar{J},(\mathrm{C} 7)$, are the analytic continuations to timelike $q^{2}$ of the indicated virtual-gluon integrals $I, J$ contributing to $F^{V x}$. While adding the soft and virtual contributions to the DY cross section, $I$ cancels $\bar{I}$ and similarly $J$ cancels $\bar{J}$. For massive off-shell one finds, for example,

$$
\bar{I}=\bar{J}=-\frac{\pi^{2}}{2} \frac{1}{\mu^{2} Q^{2}} \text {. }
$$

*Also at CERN.

†CERN Fellow.

${ }^{1}$ W. Kummer, Acta Phys. Austriaca 41, 315 (1975);

W. Konetschny and W. Kummer, Nucl. Phys. B100, 106 (1975); B108, 397 (1976); B124, 145 (1977); W. Kainz, W. Kummer, and M. Schweda, ibid. B79, 484 (1974); R. C. Crewther, in Weak and Electromagnetic Interactions at High Energy, edited by M. Levy et al. (Plenum, New York, 1976), p. 345.

${ }^{2}$ D. Amati, R. Petronzio, and G. Veneziano, Nucl. Phys. B140, 54 (1978); B146, 29 (1978); R. K. Ellis, H. Georgi, M. Machacek, H. D. Politzer, and G. G. Ross, Phys. Lett. 78B, 281 (1978); Nucl. Phys. B152, 285 (1979); S. Libby and G. Sterman, Phys. Lett. 78B, 618 (1978).

${ }^{3}$ D. J. Pritchard and W. J. Sterling, Nucl. Phys. B165, 237 (1980); G. Curci, W. Furmanski, and R. Petronzio, ibid. B175, 27 (1980); J. Kalinowski, K. Konishi, P. N. Scharbach, and T. R. Taylor, ibid. B181, 221 (1981); B181, 253 (1981).

${ }_{4}^{4}$. Frenkel and R. Meuldermans, Phys. Lett. 65B, 64 (1976).

${ }^{5}$ T. R. Taylor, Phys. Lett. 93B, 437 (1980).

${ }^{6} \mathrm{I}$. Antoniades and L. Baulieu, Harvard Report No. HUTP-80/A036 (unpublished).
${ }^{7}$ (a) B. Humpert and W. L. van Neerven, Phys. Lett. $\underline{89 B}$, 69 (1979); Nucl. Phys. B178, 498 (1981). (b) B. Humpert and W. L. van Neerven, Nucl. Phys. B184, 225 (1981).

${ }^{8}$ K. Harada, T. Kaneko, and N. Sakai, Nucl. Phys. B155, 169 (1979); B165, 545(E) (1980).

${ }^{9}$ G. T. Bodwin, C. Y. Lo, J. D. Stack, and J. D. Sullivan, Phys. Lett. 92B, 337 (1980).

${ }^{10}$ B. Humpert and W. L. van Neerven, Phys. Lett. 84B, 327 (1979); 85B, 471(E) (1979); 101B, 101 (1981).

${ }^{11}$ (a) T. Kinoshita, J. Math. Phys. $\underline{3}, 650$ (1962); (b) T. D. Lee and M. Nauenberg, Phys. Rev. 133, B1549 (1964); (c) N. Nakanishi, Prog. Theor. Phys. 19, 159 (1958).

${ }^{12}$ W. Konetschny, Nuovo Cimento 44A, 465 (1978).

${ }^{13} \mathrm{~J}$. Kubar André and F. E. Paige, Phys. Rev. D 19,221 (1979).

${ }^{14}$ G. Altarelli, R. K. Ellis, and G. Martinelli, Nucl. Phys. B157, 461 (1979).

15J. Kripfganz, Nucl. Phys. B177, 509 (1981).

${ }^{16}$ B. Humpert and W. L. van Neerven, in Proceedings of the 3rd Warsaw Symposium on Elementary Particle Physics, CERN Report No. TH-2946, 1980 (unpublished).

${ }^{17}$ D. Zwanziger, Phys. Rev. D 7,1082 (1973). 